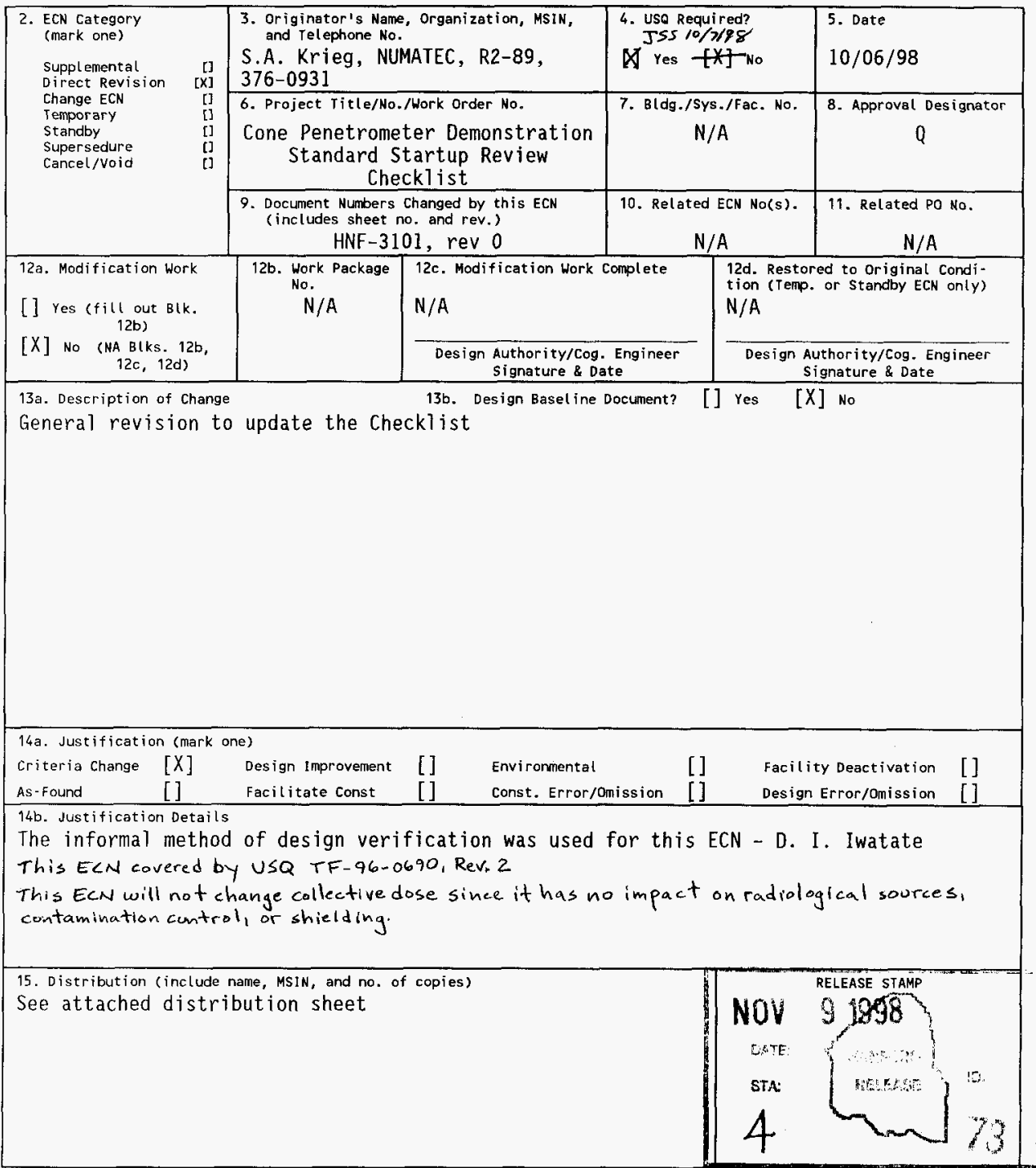




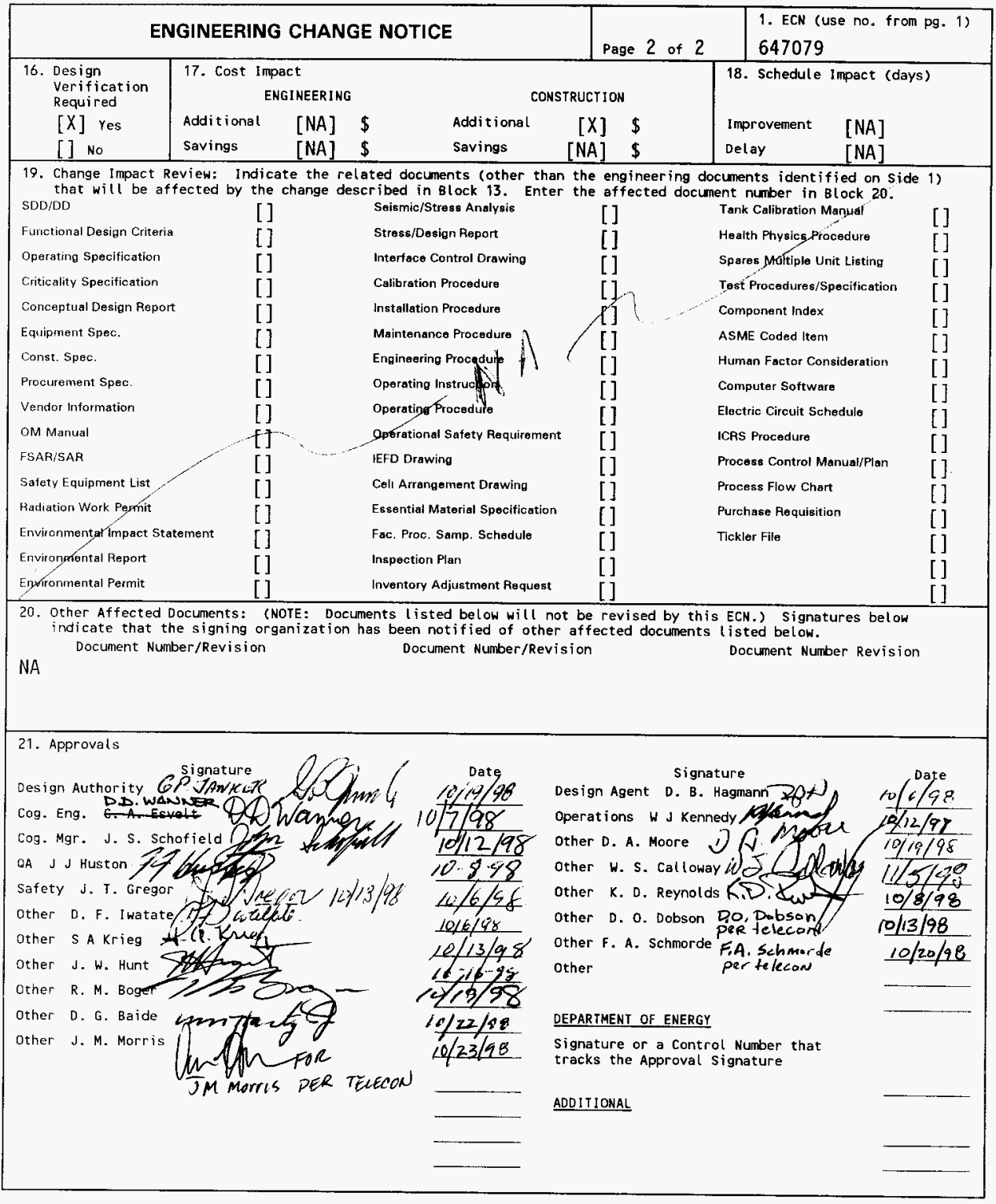


HNF-3101, Rev. 1

\section{CONE PENETROMETER DEMONSTRATION STANDARD STARTUP REVIEW CHECKLIST}

S. A. Krieg

NUMATEC Hanford Corporation, Richland, WA 99352

U.S. Department of Energy Contract DE-ACO6-96RL13200

$\begin{array}{lll}\text { EDT/ECN: } & 647079 & \text { UC: } 721 \\ \text { Org Code: } & 7 \mathrm{C} 260 & \text { Charge Code: } 103262 \\ \text { B\&R Code: } & \text { EW3130010 } & \text { Total Pages: } 4540\end{array}$

Key Words: Cone Penetrometer, Vadose, AX Farm

Abstract: Startup readiness for the Cone Penetrometer Demonstration in AX Tank Farm will be verified through the application of a Standard Startup Review Checklist. This is a listing of those items essential to demonstrating readiness to start the Cone Penetrometer Demonstration in AX Tank Farm.

ETN-97-0014

TRADEMARK DISCLAIMER. Reference herein to any specific commercial product, process, or service by trade name, trademark, manufacturer, or otherwise, does not necessarily constitute or imply its endorsement, recommendation, or favoring by the United States Government or any agency thereof or $i$ ts contractors or subcontractors.

Printed in the United States of America. To obtain copies of this document, contact: Document Control Services, P.O. Box 950, Mailstop H6-08, Richland WA 99352, Phone (509) 372-2420; Fax (509) 376-4989.
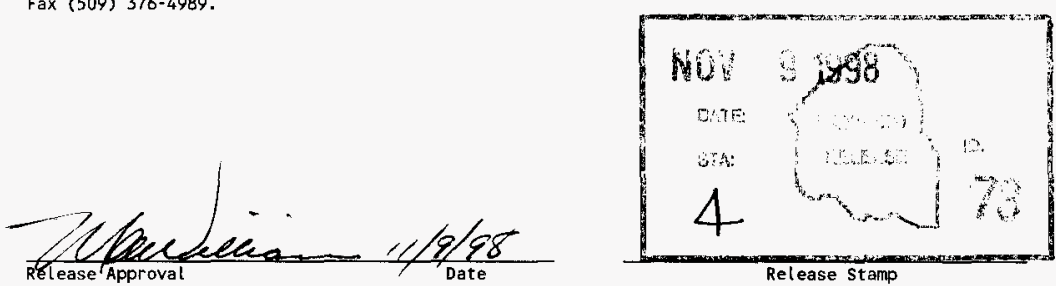

Approved for Public Release 


\section{RECORD OF REVISION}

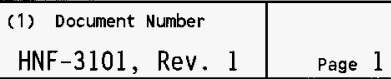

(2) Title

Cone Penetrometer Demonstration Standard Startup Review Checkl ist

CHANGE CONTROL RECORD

\begin{tabular}{|c|l|}
\hline (3) Revision & (4) Description of Change - Replace, Add, and Delete Pages \\
\hline 0 & (7) EDT-625257, 8-27-98 \\
\hline 1 RS & $\begin{array}{l}\text { General Revision to update Check1 ist, } \\
\text { per ECN-647079 }\end{array}$ \\
\hline
\end{tabular}

\begin{tabular}{|c|c|}
\hline \multicolumn{2}{|c|}{ Authorized for Release } \\
\hline (5) Cog. Engr. & (6) Cog. Mgr. \\
\hline SA Krieg & of Iyatate \\
\hline 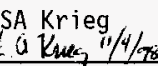 & 118 \\
\hline
\end{tabular}

$+$




\section{CONE PENETROMETER DEMONSTRATION STANDARD STARTUP REVIEW CHECKLIST}

\section{INTRODUCTION}

The Cone Penetrometer Demonstration task uses an existing piece of equipment, the Hanford Cone Penetrometer Platform (CPP), that was purchased in 1996, to push instrumented and soil sampling cone penetrometer probes into the soils adjacent to tank Ax-104. The instrumented and soil sampling probes are new designs involving state-of-the-art probe technology developed by the U.S. Army Waterways Experiment Station. Correlation of data from the instrumented probes and the soil sample lab analysis data will demonstrate the capability (or inability) of the probes to detect and measure specific constituents of the waste plume in the soil.

There is currently no identified future mission for the CPP. At the conclusion of the demonstration, the CPP will be returned to storage. The demonstration will not result in a turnover of the (existing) CPP for operations nor will it provide a CPP that is ready for additional tank farm operations.

\section{SCOPE}

The use of a standard review startup checklist to verify readiness of the CPP for safe startup and operation in the AX Tank Farm is justified by the following:

! The activity is not performed inside a waste storage tank.

! There is no inventory of radiological, chemical or hazardous material involved in the activity.

! Cone Penetrometer technology is a mature technology that has been used in private industry for decades. Truck-mounted CP systems have been used on several occasions at Hanford since the early 90's and were used most recently in 1996 to successfully deploy four Electrical Resistance Tomography (ERT) electrode array strings, to a depth of $130 \mathrm{ft}$, in 200 East area (outside the Tank Farms).

! There are permits, procedures, and hardware capabilities for the CPP that assure the probes are emplaced in the proper locations and that pipes and other underground obstructions are not contacted. These include a magnetometer to detect ferrous objects, an inclinometer to verify the maximum horizontal deviation of the probe, and a load monitoring (device on the CPP) to determine when unexpected obstructions are encountered.

! There are no physical interfaces with the Ax Farm Single-Shell Tanks or any of the Tank Farm systems. The CPP is an independently operated tool used outside the waste tanks to characterize Tank Farm soils. The only CPP/Tank Farm interface that occurs with tank farm features is when the CP probes penetrate the soil surrounding the tanks and beneath the Tank Farms. The integrity of the items within this interface will be protected by the methods described, reviewed, and approved through the Excavation Permit process.

The excavation permitting process allows selection of operational locations with appropriate consideration for aerial, surface, and submsurface 
HNE-3101

Revision 1

obstructions. The excavation permits are limited to a two month period to avoid interference with field modifications completed after the permit review. The excavation permitting process also ensures that the appropriate reviews have been completed and provides adequate assurance that the CP probe will not encounter any tank farm piping, conduit, or components. There are 12 Reviewer/approvers for the excavation pernit including the Project Engineer, Environmental, Traffic Engineer, Track Maintenance, 600 Area Landlord, Electrical utilities, Safeguards and Security, steam/water utilities, Telecommunications, Site Planning, Radiological Control, and the Cog Engineer/Facility owner. The excavation permitting process includes:

! Review of tank farms drawing, ECNs, design documentation, and work packages to establish the location of surface, aerial, and underground piping, conduits, and components.

! Discussions with tank farm operations personnel for "tribal knowledge" of tank farms that may affect the tank farm site where the cp probes will be deployed.

! The use of Ground Penetrating Radar scans to verify and chart the locations of pipes or components at the site where the cP probes will be deployed.

1 The use of a Global Positioning System to accurately locate and flag the sites where the CP probes will be deployed.

! Walk downs of the tank farm site where the CP probes will be deployed to verify the field conditions.

Based on the above, EDH and DOE RL have agreed that a standard startup review checklist is the appropriate, reasonable, and cost-effective approach to verify readiness to deploy and operate the CPP inside the AX Tank Farm boundaries.

\section{RESPONSIBILITIES}

The HTI Cone Fenetrometer Applications (D. F. Iwatate manager) organization has the funding and overall responsibility to see that all of the items on the checklist are completed. The individual assigned/obligated to complete the tasks are doing so under the auspices of HTI Cone Penetrometer Applications. The approval authority for initiation of the cone penetrometer demonstration is the Manager, Tank Waste Operations.

\section{CHECKLIST KEY}

The KEY for the responsible person initial in the checklist is shown below:

Deborah F. Iwatate

Dean B. Hagmann

Kent. D. Reynolds

William S. Callaway

Jeffrey J. Huston

Dave A. Moore

J. T. Gregor

Robert M. Boger

John $S$. Schofield

Wally J. Kennedy

John w. Hunt

Darn G. Baide

James M. Morris
Mgr., HTI Cone Penetrometer Applications

CPP Design Agent, Deployment Coordinator

Lead Geologist, Mobilization Coordinator

Analytical Support Coordinator

HTI Quality Assurance

Mgr., CPO Production Control

Mgr., TWRS Safety

Mgr., Characterization. Engineering

Mgr., Characterization Field Engineering

Mgr., Characterization Field Sampling

Mgr., Technical Basis and Planning

Mgr., DST Engineering

Mgr., Tank Operations Training 
$H N E-3101$

Revision 1

ACRONYM KEY

ARA - Applied Research Associates

BIO - Basis for Interim Operation

CAMS - Computer Aided Management System

$\mathrm{CP}$ - Cone Penetrometer

CPP - Cone Penetrometer Platform

DTS - Deficiency Tracking system

HATS - Hanford Action Tracking System

HGET - Hanford General Education Training

HPT - Health Physics Technician

HTI - Hanford Tanks Initiative

JCS - Job Control System

JHA/JSA - Job Hazards Analysis / Job Safety Analysis

NCR - Non Conformance Report

NOC - Notice of Construction

PHA - Preliminary Hazards Analysis

RPR - Radiological Problem Report

RWP - Radiological Work Permit

WDOH - Washington Department of Health

USQ - Unreviewed Safety Question

XRF - X-Ray Fluorescence 
HNE -3101

Revision 1

Table of Contents

\section{0 Maintenance}

$\begin{array}{ll}\text { 1.1 Maintenance Program Structure \& Management } \\ \text { 1.2 Maintenance Equipment, Facilities \& Technology } \\ \text { 1.3 Work Identification, Planning \& Scheduling } \\ \text { 1.3.1 JCS Work Packages } \\ \text { 1.3.2 Plant Forces Work Review } \\ \text { 1.4 Maintenance Procedures } \\ 1.5 \text { Maintenance Information } \\ 1.6 & \text { Maintenance Quality Assurance } \\ 1.7 & \text { Preventive Maintenance \& Evaluation Programs } \\ 1.8 & \text { Calibration Program } \\ 1.9 & \text { Maintenance Outage Program }\end{array}$

Page

.o Environment, Health \& Safety 10

2.1 General 10

2.2 Reactor/Facility Safety Review \& Analysis 11

2. 3 Response to Design Basis Accidents 11

$\begin{array}{ll}2.4 & \text { Industrial Hygiene } \\ 2.5\end{array}$

$\begin{array}{ll}2.5 \text { Radiation Safety } & 12 \\ 2.6 & 12\end{array}$

2.6 Nuclear Safety/Criticality Safety 13

2.7 Dccupational Safety 13

2.8 Fire Protection \& Life Safety 14

2.9 CPP Transportation Safety 14

2.10 CP Probe Transportation Safety 15

2.11 Environmental Protection 15

2.12 Emergency Preparedness 15

2.13 Safety Systems \& Equipment 16

2.14 Environmental Qualification 16

$\begin{array}{ll}2.15 & \text { Adverse Weather Protection } \\ 2.16 & 16\end{array}$

2.16 Chemical Process Safety 16

3.0 Services 17

3.1 Laboratory Analysis 17

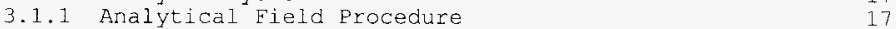

3.1.2 AX-104 Vadose Zone Sampling and Analysis Plan 17

$\begin{array}{ll}3.2 \text { Safeguards } & 17\end{array}$

$\begin{array}{ll}3.3 \text { Security } & 17\end{array}$

$\begin{array}{ll}3.4 \text { Transportation } & 18\end{array}$

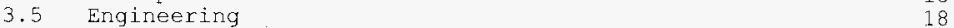

3.5.1 Engineering Task Plan 1.8

3.5.2 AX-104 Dome Loading Evaluation 18

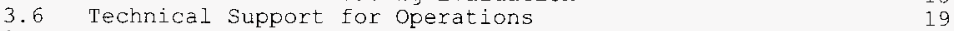

3.7 Research \& Development Support 19

$\begin{array}{ll}3.8 \text { Waste Management (Waste Disposal Requirements) } & 20\end{array}$

$\begin{array}{ll}3.9 \text { Utilities } & 20\end{array}$

4. 0 Controls and Program Functioning 20

$\begin{array}{lll}4.1 & \text { Safety Analysis } & 20\end{array}$

$\begin{array}{ll}4.2 & \text { Process Hazards Reviews }\end{array}$

4.3 Internal \& External Communications 21

$\begin{array}{ll}4.4 \text { Permits } & 21\end{array}$

4.4.1 Excavation Permit for CE Probe Deployment 21

4.4.2 WDOH NOC

4.5 Materials Control \& Accountability 22

4.6 Organization, Responsibilities \& Authority 22

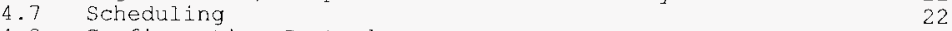

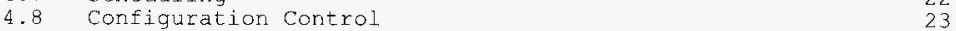


4.9 Technical Control \& Data

4.9.1 CP Grouting Strategy Document

Page

4.9.2 Radiation Risk Assessment Document 24

4.10 Facility Ferformance Measurement \& Evaluation 24

4.11 Analysis of Startup of Facility Operation \& Programmatic 24 Aspects

4.12 Program Definition \& Goal Realization 24

\section{0 Regulatory Compliance}

5.1 DOF FS\&H Orders

5.2 Codes and Standards 25

5.3 Environmental Reviews 26

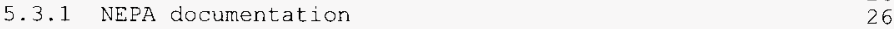

5.3.2 WDOH Air Permitting Review 26

$\begin{array}{lll}5.4 & \text { Statutory Requirements } & 27\end{array}$

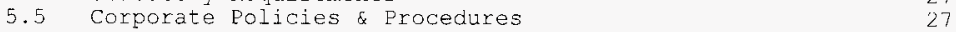

6.0 Structures \& Hardware 28

$\begin{array}{lll}6.1 \text { Design Program } & 28\end{array}$

$\begin{array}{ll}6.2 \text { Materials Control } & 28\end{array}$

6.3 Construction Program 28

6.4 Structures 29

6.5 Primary Process Systems \& Equipment 29

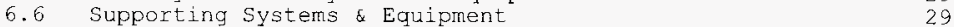

6.7 Special Equipment 30

6.8 Process, Facility \& Site Interfaces 30

6.9 Materials, Spare Parts, \& Spare Equipment 30

6.10 Adverse Weather Protection $\quad 31$

\subsection{Personnel}

$\begin{array}{lll}7.1 & \text { Personnel Selection } & 31 \\ 7.2 & \text { Training \& Skill Verification } & 31 \\ 7.2 .1 \text { Training Plan } & 31 \\ 7.2 .2 \text { Verification } & 31 \\ 7.3 & \text { Knowledge \& Competence } & 32 \\ 7.4 & \text { Adherence to Proper Practice } & 32 \\ 7.5 & \text { Staffing Levels } & 33 \\ 7.6 & \text { Recertification } & 33 \\ 7.7 & \text { Oral/Written Boards } & 33\end{array}$

\section{0 Operations}

8.1 Operations Structure \& Management 33

8.2 Pperations Procedures 34

8.2.1 General Operating Description 34

8.2.2 CPP Move and Set-up Work Instruction 34

8.2.3 Multi-Sensor Probe Operating Instructions 34

8.2. 4 Soil Sample Retrieval Work Instruction 34

8.2.5 CP Grouting Procedure 35

8.2.6 Skid Move Work Instruction 35

8.2.7 CPP Operating Instructions 35

8.3 Operations Information. 36

8.4 Operating Practices 36

8.5 Control of Systems and Equipment 36

$\begin{array}{ll}\text { 8.6 Operations Materials \& Supplies } & 37\end{array}$

8.7 Experimental Operations 37

$\begin{array}{ll}8.8 \text { Operations Quality Assurance } & 37\end{array}$

\subsection{Quality Assurance}

9.1 OA Program Implementation $\quad 38$ 


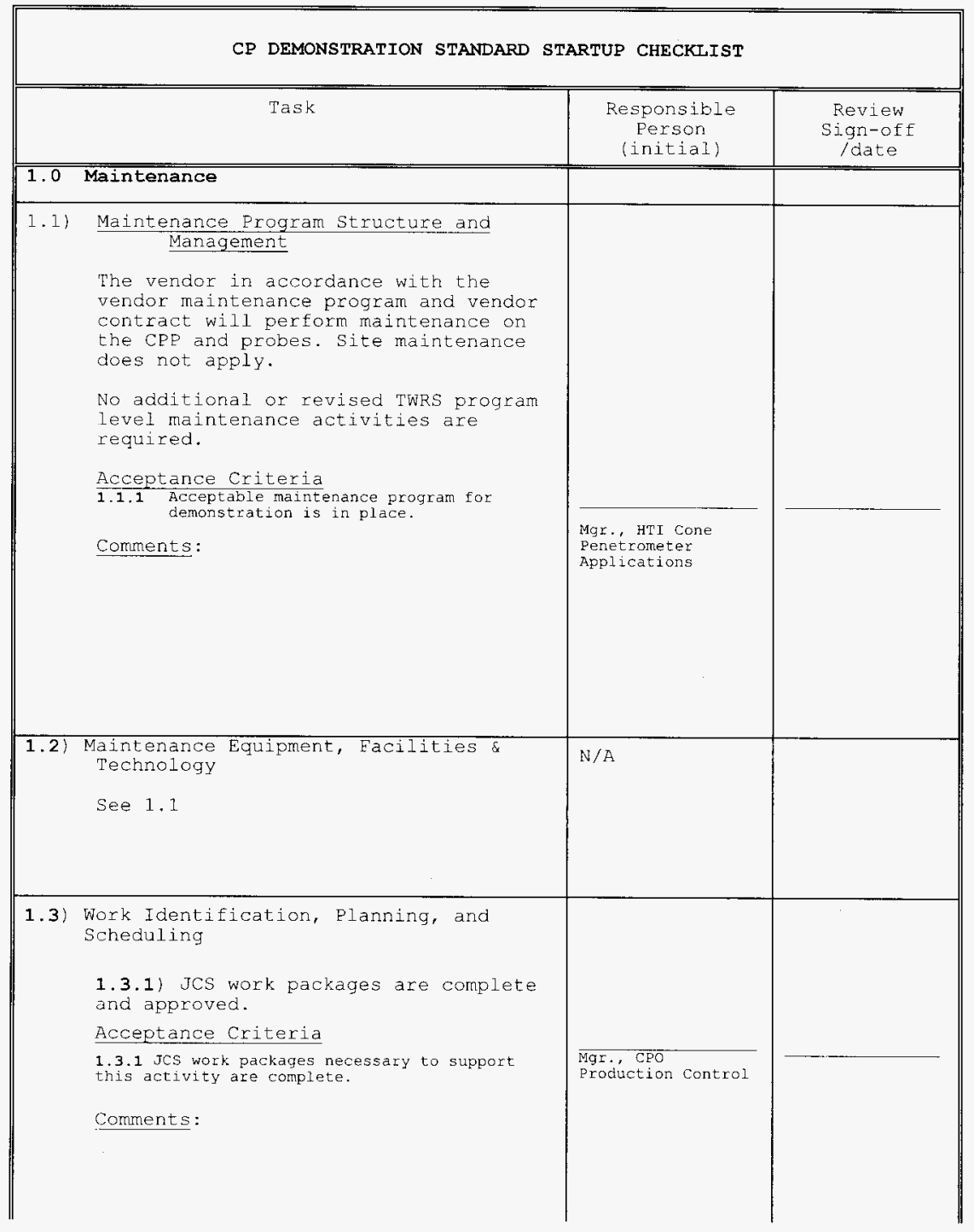


CP DEMONSTRATION STANDARD STARTUP CHECKIIST

\begin{tabular}{|c|c|c|c|}
\hline & Task & $\begin{array}{c}\text { Responsible } \\
\text { Person } \\
\text { (initial) }\end{array}$ & $\begin{array}{c}\text { Review } \\
\text { Sign-off } \\
\text { /date }\end{array}$ \\
\hline & $\begin{array}{l}\text { 1.3.2) Plant Eorces work Review (PEWR) } \\
\text { is complete. } \\
\text { HNF-PRo-70 does not reference } \\
\text { technology demonstrations as needing a } \\
\text { EFWR. This activity will not involve } \\
\text { construction, modification or repair } \\
\text { work and is not a construction } \\
\text { project. } \\
\text { Acceptance Criteria } \\
\text { 1.3.2 pewR necesary for this activity is } \\
\text { complete. } \\
\text { Comments: }\end{array}$ & $\begin{array}{l}\text { Mgr., CPO } \\
\text { Production Control }\end{array}$ & \\
\hline 1.4) & $\begin{array}{l}\text { Maintenance Procedures } \\
\text { Vendor maintenance schedules for the } \\
\text { cone Penetrometer platform already } \\
\text { exist (they were provided as part of } \\
\text { the cPP procurement). Plant } \\
\text { maintenance procedures are not } \\
\text { required since the cPP will not be an } \\
\text { operational piece of equipment at the } \\
\text { completion of the demonstration. The } \\
\text { CP probes associated with the HTI } \\
\text { demonstration are one of a kind } \\
\text { demonstration tools that require no } \\
\text { maintenance. They exist for the } \\
\text { duration of the task. } \\
\text { Acceptance criteria } \\
\text { l.4.1 The vendor mantenance program for the } \\
\text { cone penetrometer Platform have been Ieviewed } \\
\text { and found acceptable for the ce demonstration. } \\
\text { Comments }\end{array}$ & $\begin{array}{l}\text { Let HT Cone } \\
\text { Mgr, HTI } \\
\text { Penetrometer } \\
\text { Applications }\end{array}$ & \\
\hline
\end{tabular}




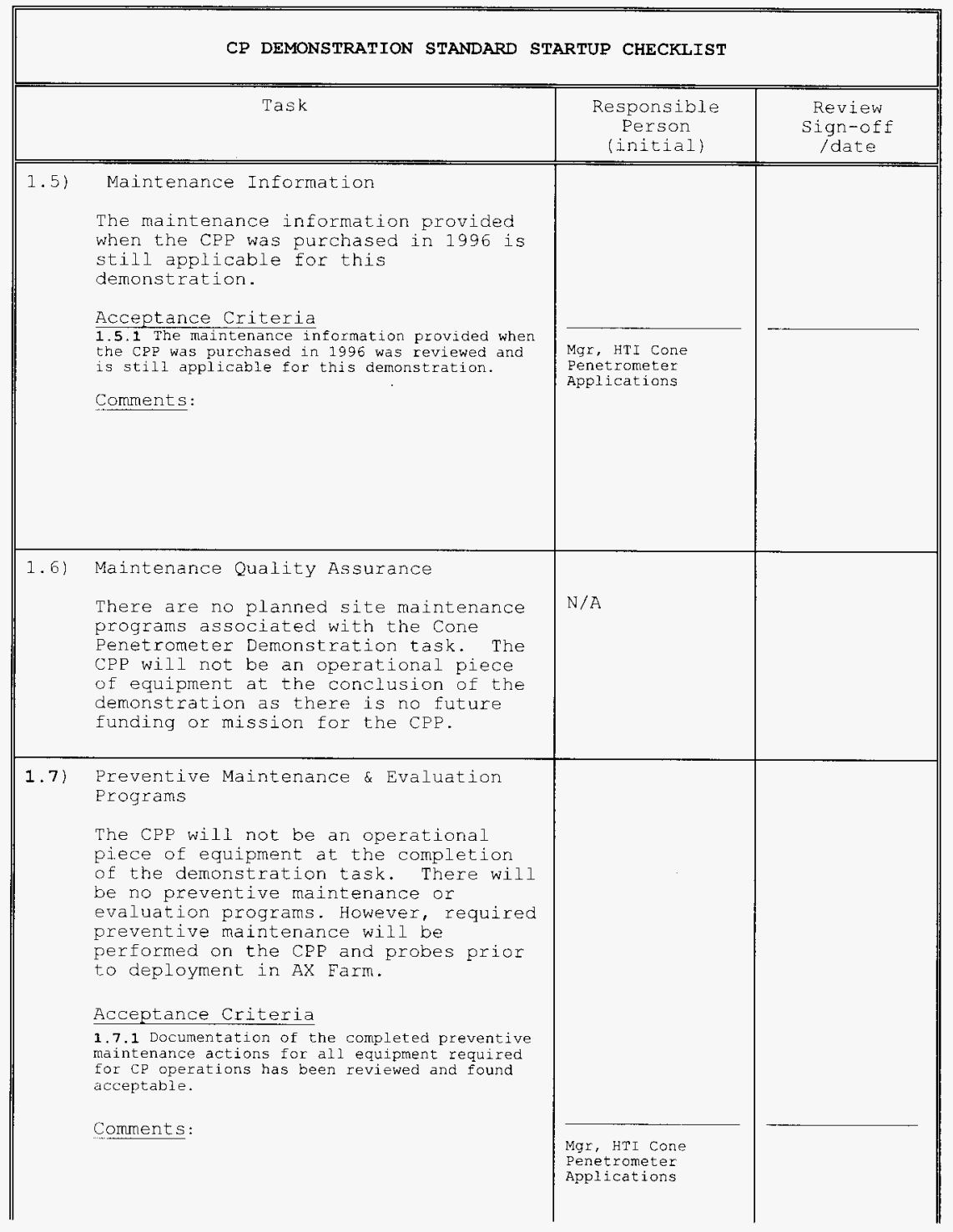




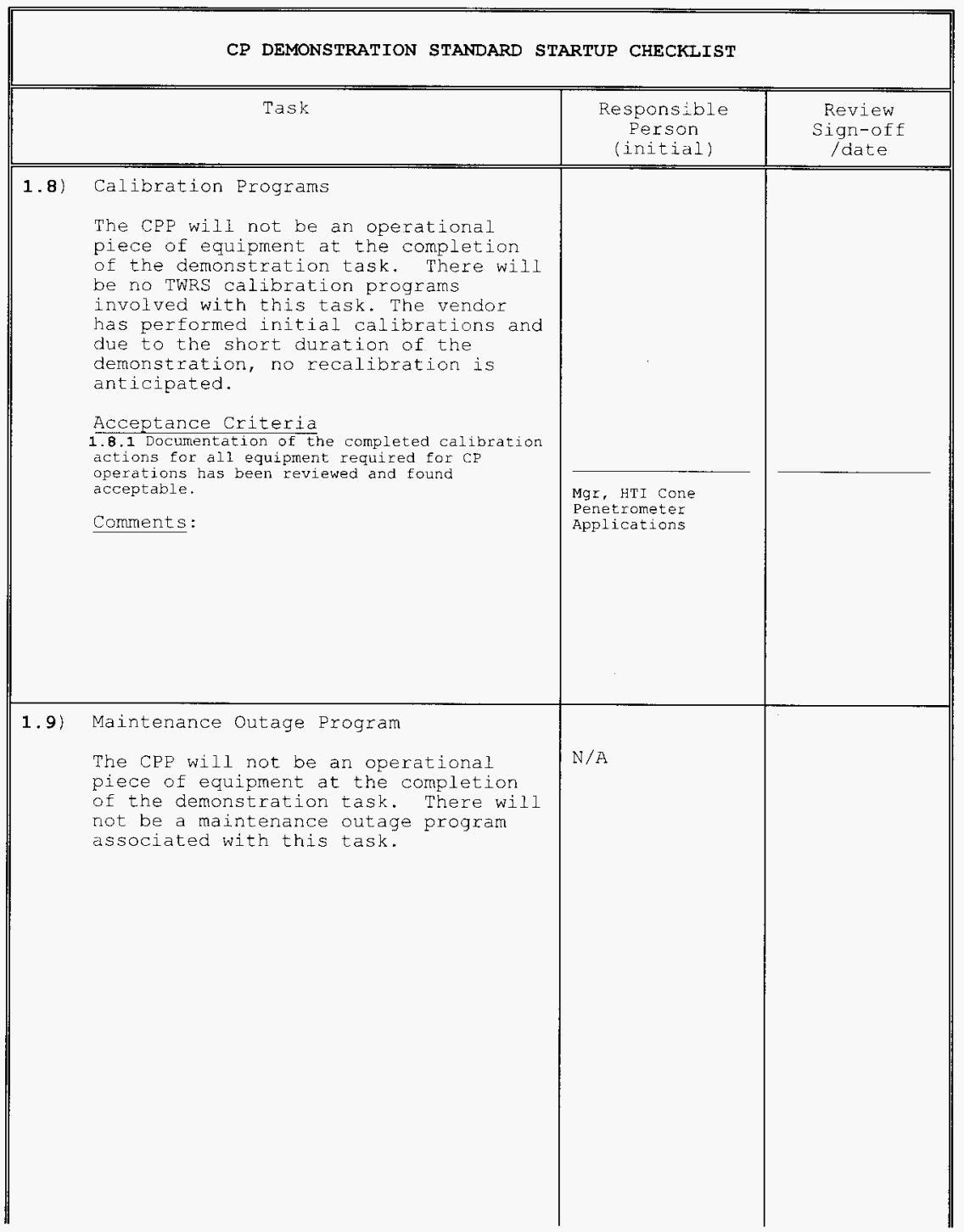




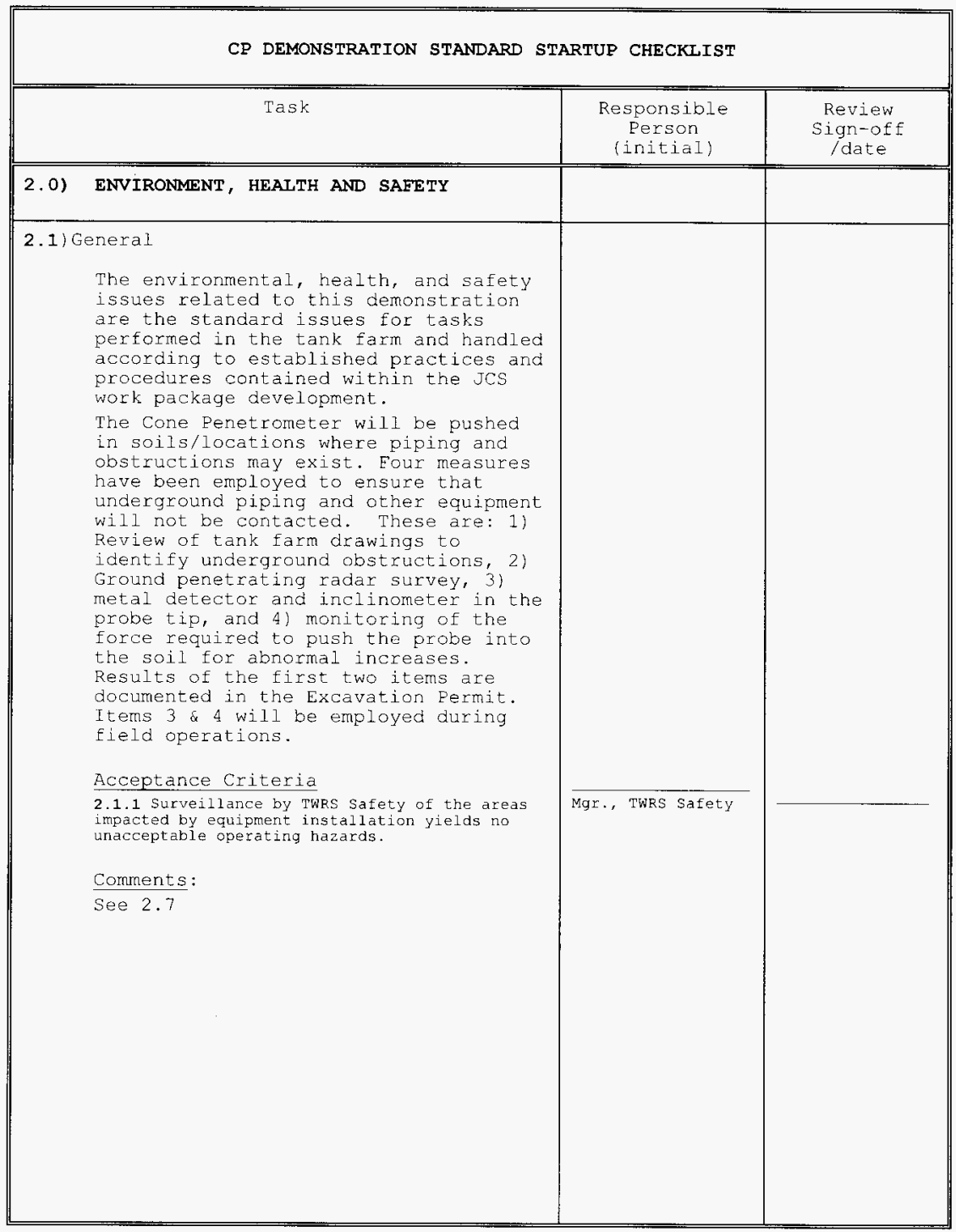




\begin{tabular}{|c|c|c|c|}
\hline \multicolumn{4}{|c|}{ CP DEMONSTRATION STANDARD STARTUP CHECKLIST } \\
\hline & Task & $\begin{array}{c}\text { Responsible } \\
\text { Person } \\
\text { (initial) }\end{array}$ & $\begin{array}{c}\text { Review } \\
\text { Sign-off } \\
\text { /date }\end{array}$ \\
\hline 2.2$)$ & $\begin{array}{l}\text { Reactor/Eacility Safety Review and } \\
\text { Safety Analysis } \\
\text { The CP demonstration task was USQ } \\
\text { screened (USQ TE-97-0876) and the } \\
\text { activity was found to be bound by the } \\
\text { authorization basis. The USQ screen } \\
\text { was based on a Preliminary Hazard } \\
\text { Analysis (PHA) which was surmarized in } \\
\text { HNE-SD-WM-HIE-012. No additional } \\
\text { safety analysis is required. } \\
\text { Acceptance Criteria } \\
\text { 2.2. USR screenings for the ce demonstration } \\
\text { andior evaluations supporting equipment } \\
\text { installation and required equipment added to } \\
\text { support CP operations are complete. } \\
\text { Comments: }\end{array}$ & 更 & . \\
\hline 2.3) & $\begin{array}{l}\text { Response to Design Basis Accidents } \\
\text { No design basis accidents were found } \\
\text { to be applicable in the PHA. } \\
\text { Acceptance criteria } \\
\frac{2.3 .1 \text { A Hazards Identification \& Evaluation }}{\text { document and FHA was summarized in HNF-SD-wM- }} \\
\text { HI-012 and the hazards are acceptable. } \\
\text { Comments: }\end{array}$ & $\begin{array}{l}\text { Mgr, HTI Cone } \\
\text { Penetrometer } \\
\text { Applications }\end{array}$ & \\
\hline
\end{tabular}




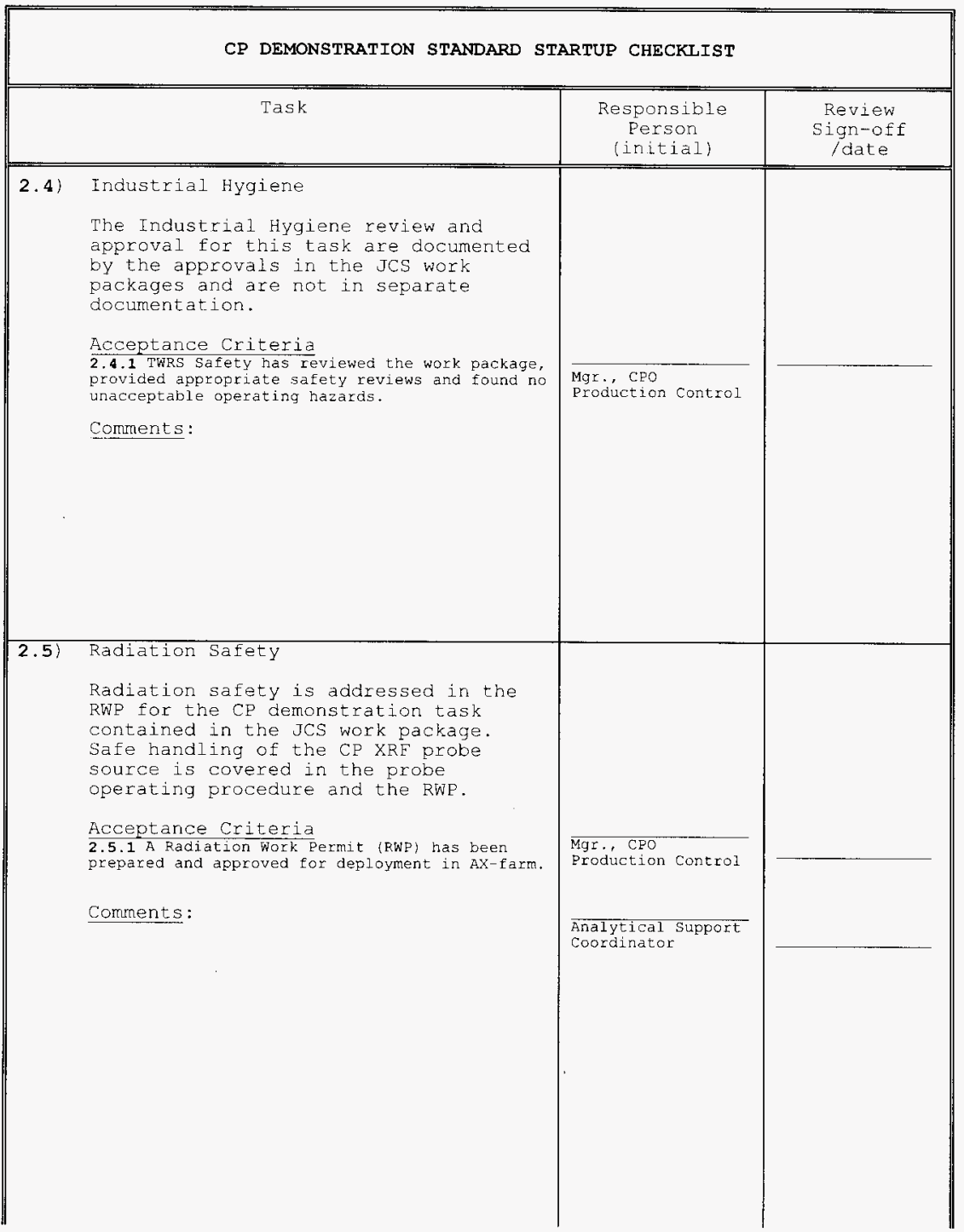




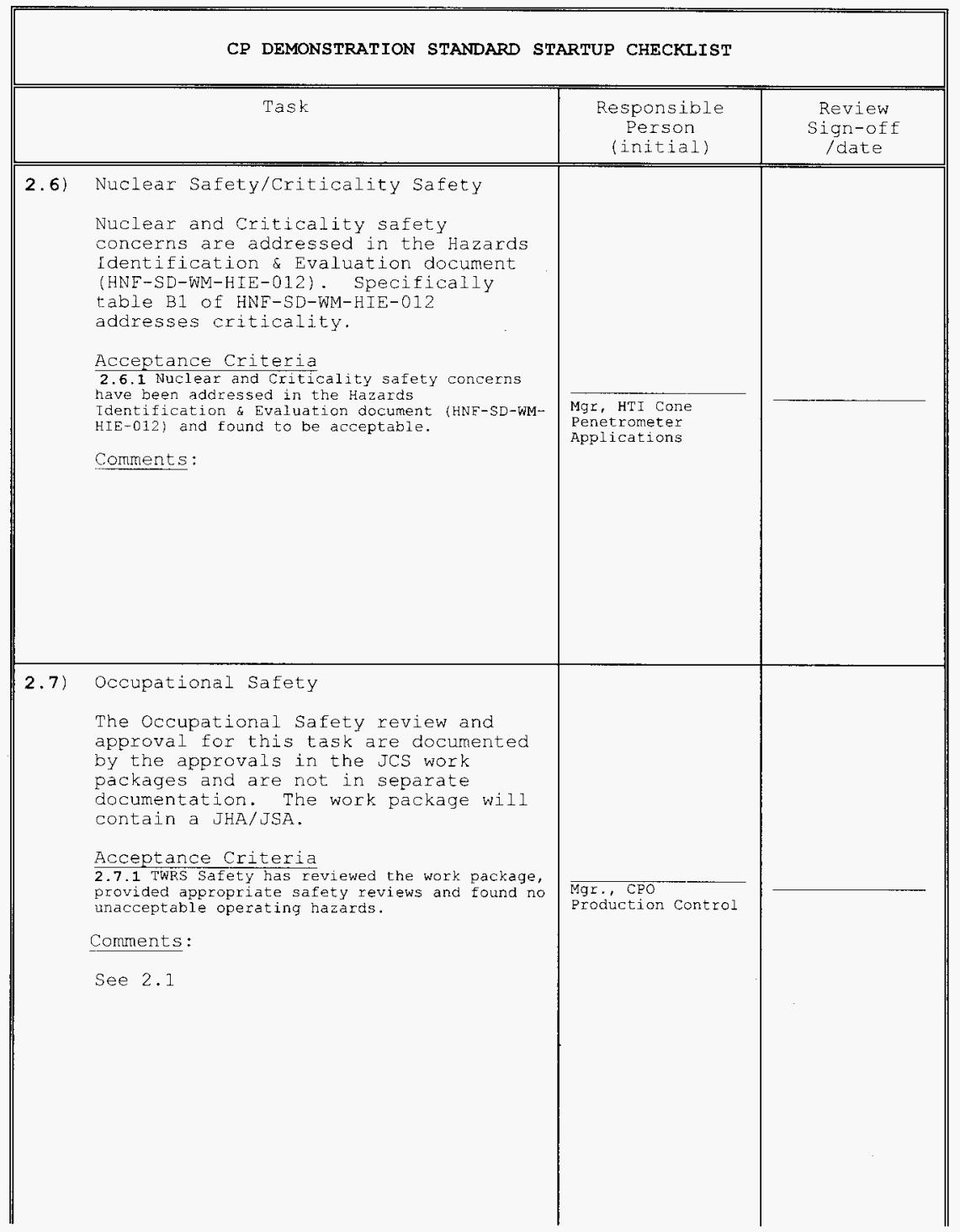




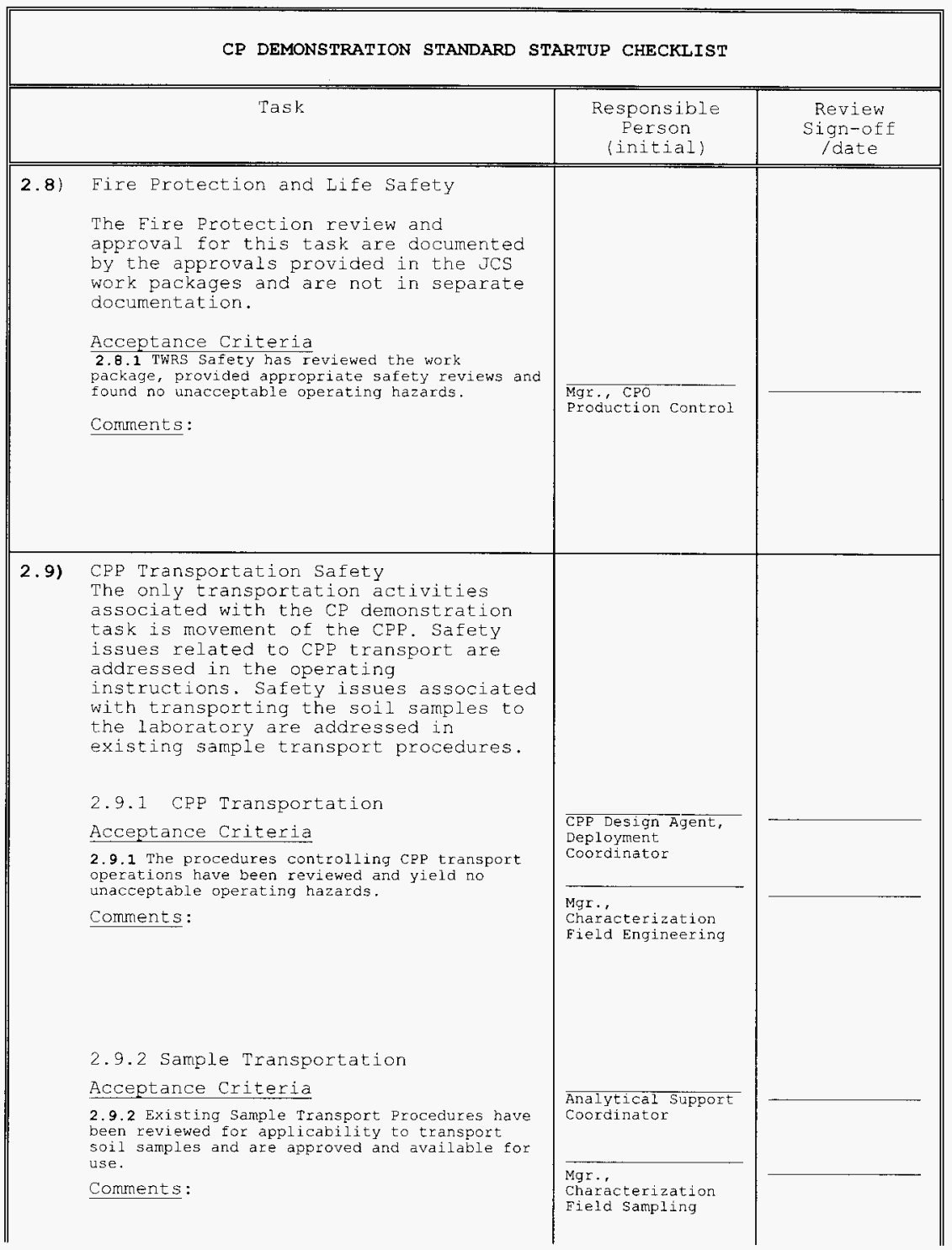




\section{CP DEMONSTRATION STANDARD STARTUP CHECKLIST}

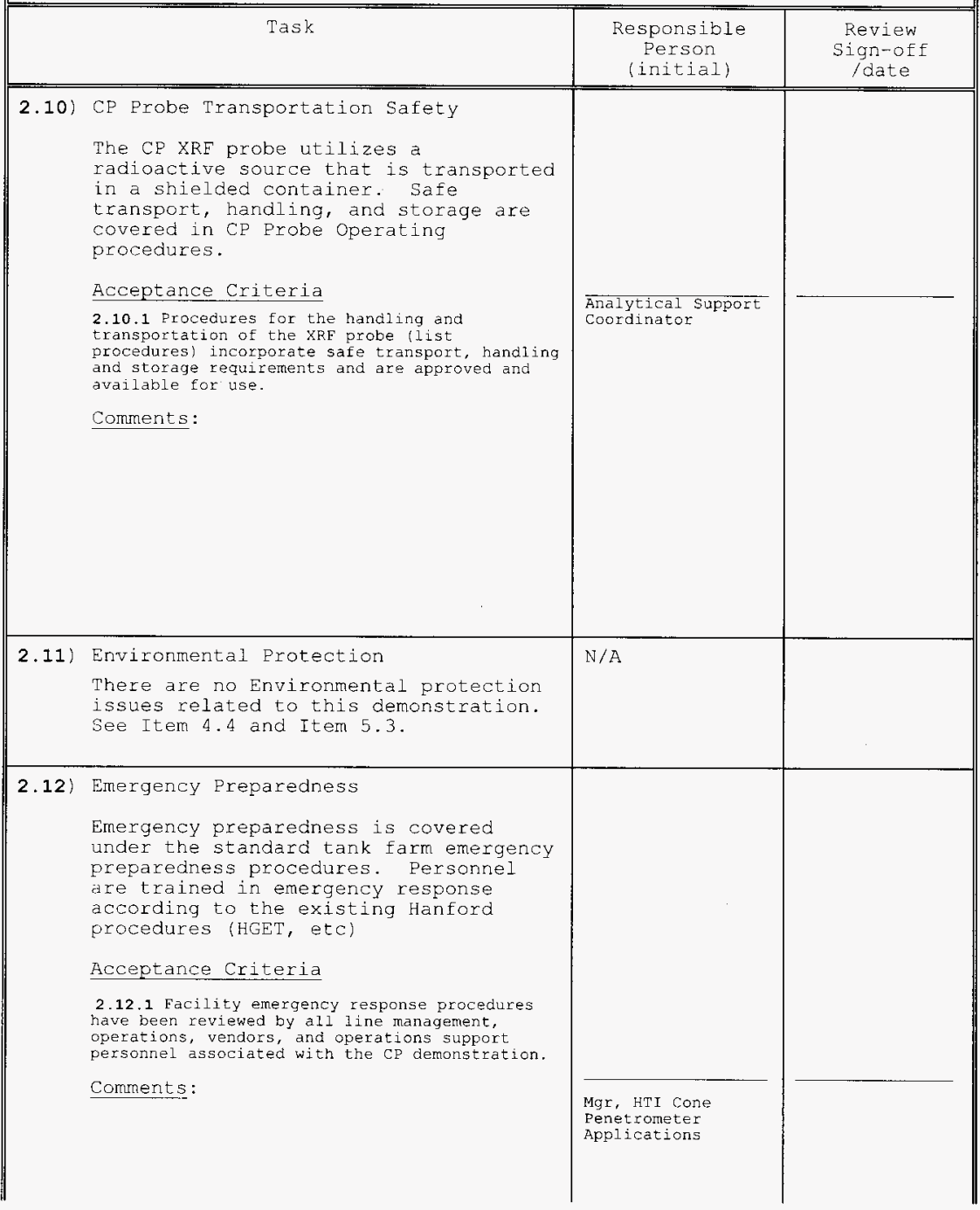


CP DEMONSTRATION STANDARD STARTUP CHECKLIST

\begin{tabular}{|c|c|c|c|}
\hline \multicolumn{2}{|c|}{ Task } & \multirow{2}{*}{$\begin{array}{l}\begin{array}{c}\text { Responsible } \\
\text { Person } \\
\text { (initial) }\end{array} \\
\text { N/A }\end{array}$} & \multirow{2}{*}{$\begin{array}{c}\text { Review } \\
\text { Sign-off } \\
\text { /date } \\
\end{array}$} \\
\hline 2.13$)$ & $\begin{array}{l}\text { Safety systems and Equipment } \\
\text { There are no safety class or safety } \\
\text { significant ssCs associated with this } \\
\text { demonstration. There are no safety } \\
\text { systems or equipment on the cFP. }\end{array}$ & & \\
\hline 2.14$)$ & $\begin{array}{l}\text { Environmental Qualification } \\
\text { There are no environmental } \\
\text { qualifications associated with this } \\
\text { demonstration. }\end{array}$ & $\mathrm{N} / \mathrm{A}$ & \\
\hline 2.15$)$ & $\begin{array}{l}\text { Adverse weather Protection } \\
\text { The CPE was designed for outdoor } \\
\text { storage. The Cone Penetrometer } \\
\text { demonstration will not be conducted in } \\
\text { adverse weather conditions. In } \\
\text { accordance with standard tank farm } \\
\text { procedures, operations will be shut } \\
\text { down for excessive wind, lightning, } \\
\text { etc. } \\
\text { Acceptance Criteria } \\
\text { 2.ms.l operating limits and parameters } \\
\text { established, document on file. } \\
\text { Comments: }\end{array}$ & $\begin{array}{l}\text { Mgr., } \\
\text { Characterization } \\
\text { Field Engineering }\end{array}$ & \\
\hline 2.16$)$ & $\begin{array}{l}\text { Chemical Process Safety } \\
\text { There are no chemical processes } \\
\text { associated with the cPP or with this } \\
\text { demonstration in tank farms. }\end{array}$ & $N / A$ & \\
\hline
\end{tabular}


CP DEMONSTRATION STANDARD STARTUP CHECKLIST

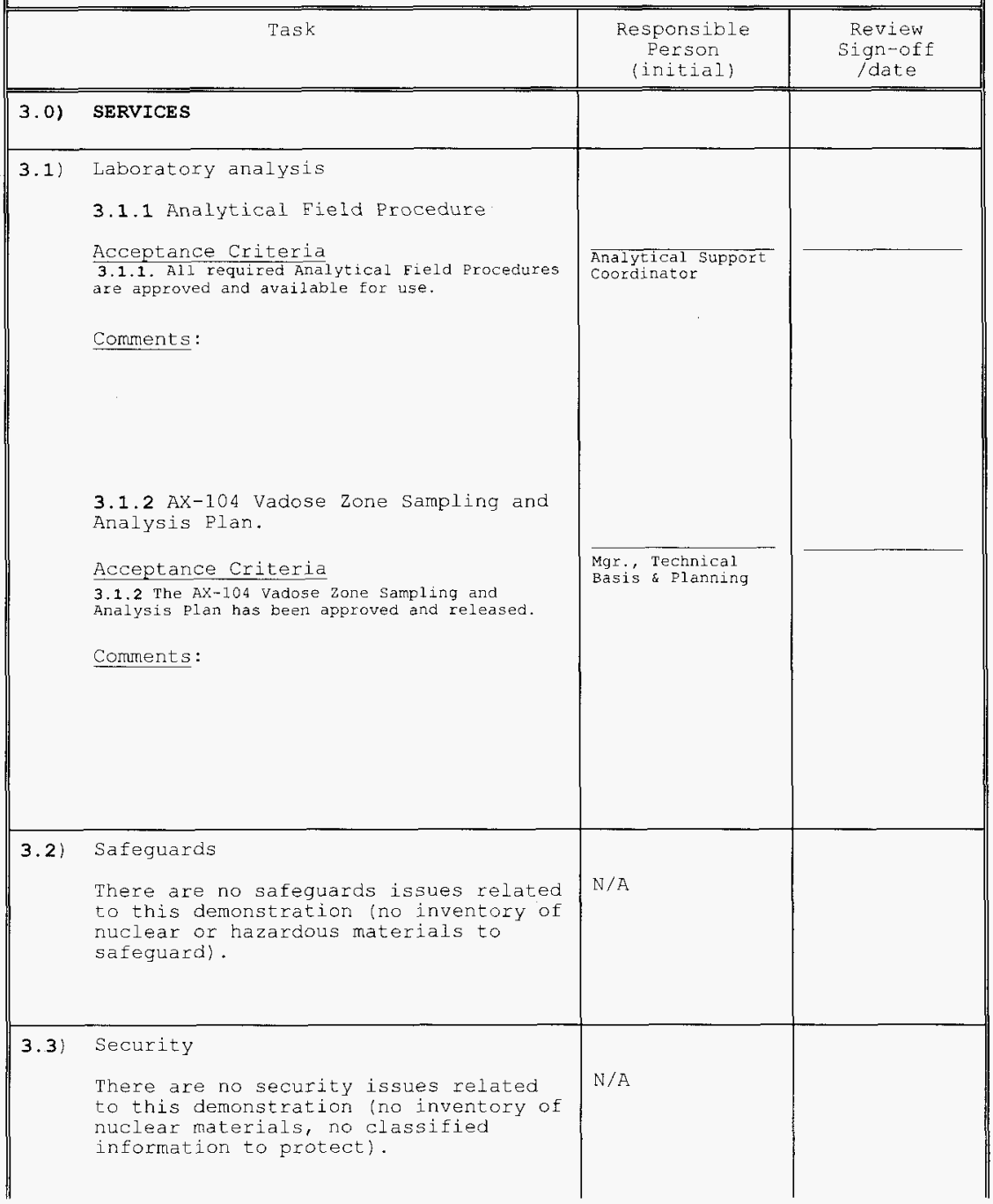




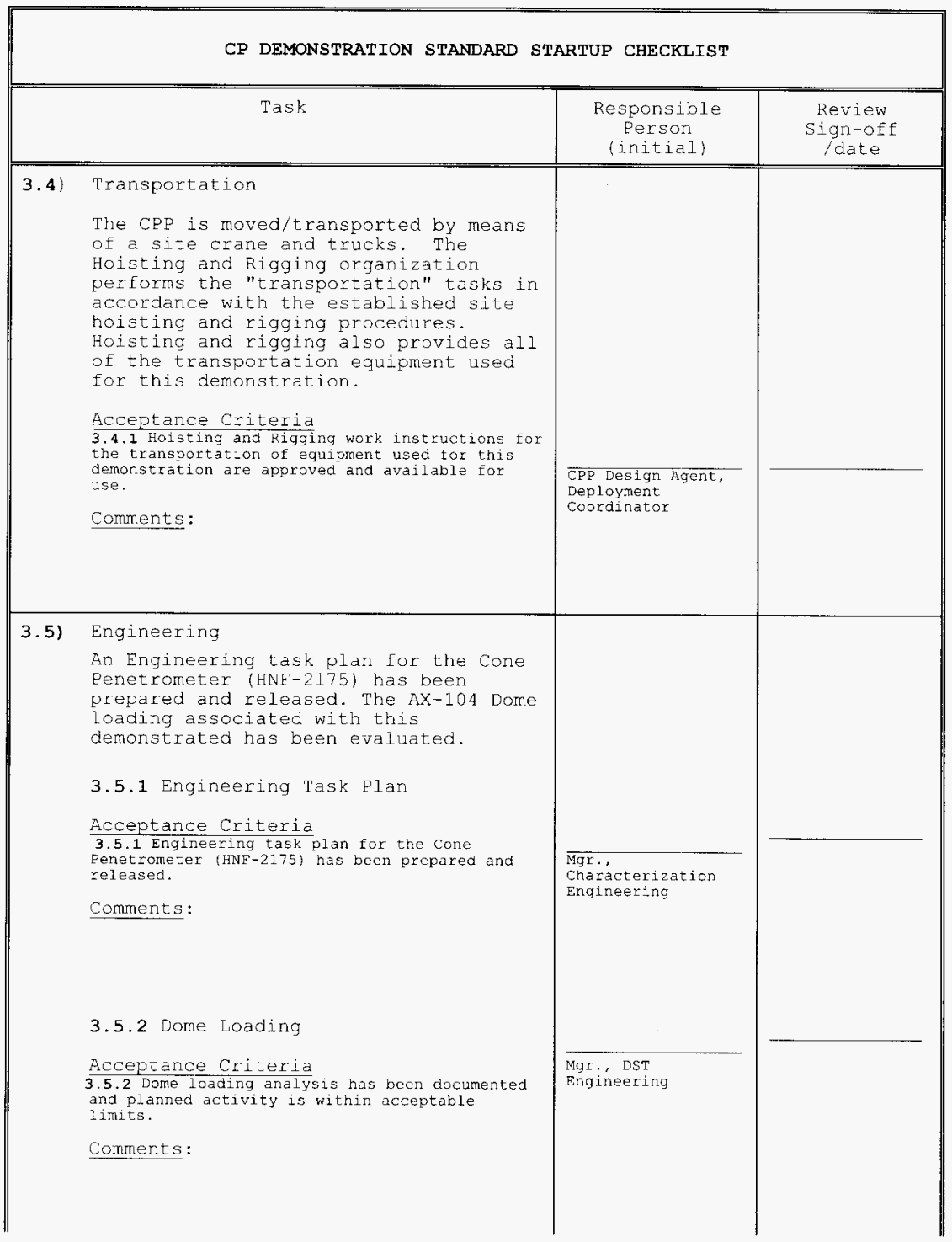




\section{CE DEMONSTRATION STANDARD STARTUP CHECKLIST}

\begin{tabular}{|c|c|c|c|}
\hline & Task & $\begin{array}{c}\text { Responsible } \\
\text { Person } \\
\text { (initial) }\end{array}$ & $\begin{array}{l}\text { Review } \\
\text { Sign-off } \\
\text { /date }\end{array}$ \\
\hline 3.6$)$ & $\begin{array}{l}\text { Technical Support of operations } \\
\text { TwRs technical. support for the } \\
\text { demonstration will be provided by the } \\
\text { cog engineer, design authority, and } \\
\text { PIC. } \\
\text { Acceptance Criteria } \\
\text { 3.6.1 Adequate technical support has been } \\
\text { identified and is available. } \\
\text { Comments: }\end{array}$ & $\begin{array}{l}\overline{M g r} ., \\
\text { Characterization } \\
\text { Engineering }\end{array}$ & \\
\hline 3.7$)$ & $\begin{array}{l}\text { Research and Development Support } \\
\text { There are no research and development } \\
\text { activities associated with this task. } \\
\text { This task involves the use of existing } \\
\text { technologies and methods within a } \\
\text { operational environment for the } \\
\text { purpose of demonstration and } \\
\text { evaluation. No research and } \\
\text { development is performed during this } \\
\text { work. Existing instruments and field } \\
\text { tools are combined, reconfigured, and } \\
\text { used in a unique way to address } \\
\text { deployment location physical } \\
\text { constraints and information/data } \\
\text { needs. Data provided by instruments } \\
\text { and samples during this work will be } \\
\text { used to supplement other information } \\
\text { for the use in programmatic planning } \\
\text { and decision-making. The quality of } \\
\text { data and the deployment experience } \\
\text { will be evaluated for possible future, } \\
\text { routine use and applications. A data } \\
\text { quality objective (Doo) and a sampling } \\
\text { and analysis plan (SAP) has been } \\
\text { prepared to guide task planning and } \\
\text { technology preparation toward } \\
\text { appropriate and needed goals. } \\
\text { Eollowing the guidance of a Doo and } \\
\text { sap also provides credibility and } \\
\text { relevance to the deployment experience } \\
\text { and the data/samples that are } \\
\text { obtained. }\end{array}$ & $N / A$ & \\
\hline
\end{tabular}




\section{CP DEMONSTRATION STANDARD STARTUP CHECKLIST}

\begin{tabular}{|c|c|c|c|}
\hline & Task & $\begin{array}{c}\text { Responsible } \\
\text { Person } \\
\text { (initial) }\end{array}$ & $\begin{array}{c}\text { Review } \\
\text { Sign-off } \\
\text { /date }\end{array}$ \\
\hline 3. 8$)$ & $\begin{array}{l}\text { Waste Management } \\
\text { Waste disposal requirements has been } \\
\text { developed to establish the methods of } \\
\text { handling contaminated wastes generated } \\
\text { by this demonstration. These } \\
\text { requirements are included within the } \\
\text { JCs work package development. } \\
\text { Acceptance Criteria } \\
\text { 3.8.1 Waste disposal requirements for handling } \\
\text { contaminated wastes generated by this Jcs work } \\
\text { demonstration are ircluded within the Jcs wo. } \\
\text { Comments: }\end{array}$ & $\begin{array}{l}. \\
\text { Mgr., } \\
\text { Characterization } \\
\text { Field Sampling }\end{array}$ & \\
\hline 3.9$)$ & $\begin{array}{l}\text { Utilities } \\
\text { The CPP is a self-contained system } \\
\text { that does not require external } \\
\text { utilities. }\end{array}$ & $\mathrm{N} / \mathrm{A}$ & \\
\hline 4.0$)$ & CONTROLS AND PROGRAM FUNCTIONING & & \\
\hline 4.1$)$ & $\begin{array}{l}\text { Safety Analysis } \\
\text { The CP demonstration task was USQ } \\
\text { screened (USQ TE-97-0876) and the } \\
\text { activity was found to be bounded by } \\
\text { the authorization basis. The USQ } \\
\text { screen was based on a PHA, which was } \\
\text { summarized in HNE-SD-WM-HIE-012. No } \\
\text { additional safety analysis is } \\
\text { required. } \\
\text { Comments: } \\
\text { Combined with } 2.2 .1\end{array}$ & $\begin{array}{l}\text { Mgr, HTI Cone } \\
\text { Penetrometer } \\
\text { Applications }\end{array}$ & \\
\hline
\end{tabular}


CP DEMONSTRATION STANDARD STARTUP CHECKIIST

\begin{tabular}{|c|c|c|c|}
\hline \multicolumn{2}{|c|}{ Task } & \multirow{2}{*}{$\begin{array}{c}\text { Responsible } \\
\text { Person } \\
\text { (initial) }\end{array}$} & \multirow{2}{*}{$\begin{array}{c}\text { Review } \\
\text { Sign-off } \\
\text { /date }\end{array}$} \\
\hline 4.2) & $\begin{array}{l}\text { Process Hazards Reviews } \\
\text { A PHA was performed and summarized in } \\
\text { HNF-SD-WM-HIE-012. This satisfies the } \\
\text { requirement for a Frocess Hazards } \\
\text { Review. } \\
\text { Comments: } \\
\text { Combined with } 2.3 .1\end{array}$ & & \\
\hline 4.3$)$ & $\begin{array}{l}\text { Internal \& External Communications } \\
\text { CPP does not incorporate any } \\
\text { communications equipment. CPP } \\
\text { operations occur around the unit with } \\
\text { all workers/operations within eye and } \\
\text { ear range of each other. } \\
\text { Comments: }\end{array}$ & $\mathrm{N} / \mathrm{A}$ & \\
\hline 4.4) & $\begin{array}{l}\text { Permits } \\
\text { All applicable environmental } \\
\text { requirements are identified. } \\
\text { Applicable environmental permits } \\
\text { and/or approvals are received and } \\
\text { requirements are implemented in the } \\
\text { appropriate procedures/documents as } \\
\text { necessary } \\
\text { 4.4.1 Excavation permit } \\
\text { Acceptance Criteria } \\
\mathbf{4 . 4 . 1} \text { An Excavation permit (including the } \\
\text { cultural resources review) for deployment of the } \\
\text { hane penetrometer probes into the tank farm soil } \\
\text { Com been approved. } \\
\text { Comments: }\end{array}$ & $\begin{array}{l} \\
\\
\text { Mead Geologist, } \\
\text { Coordization }\end{array}$ & \\
\hline
\end{tabular}


CP DEMONSTRATION STANDARD STARTUP CHECKLIST

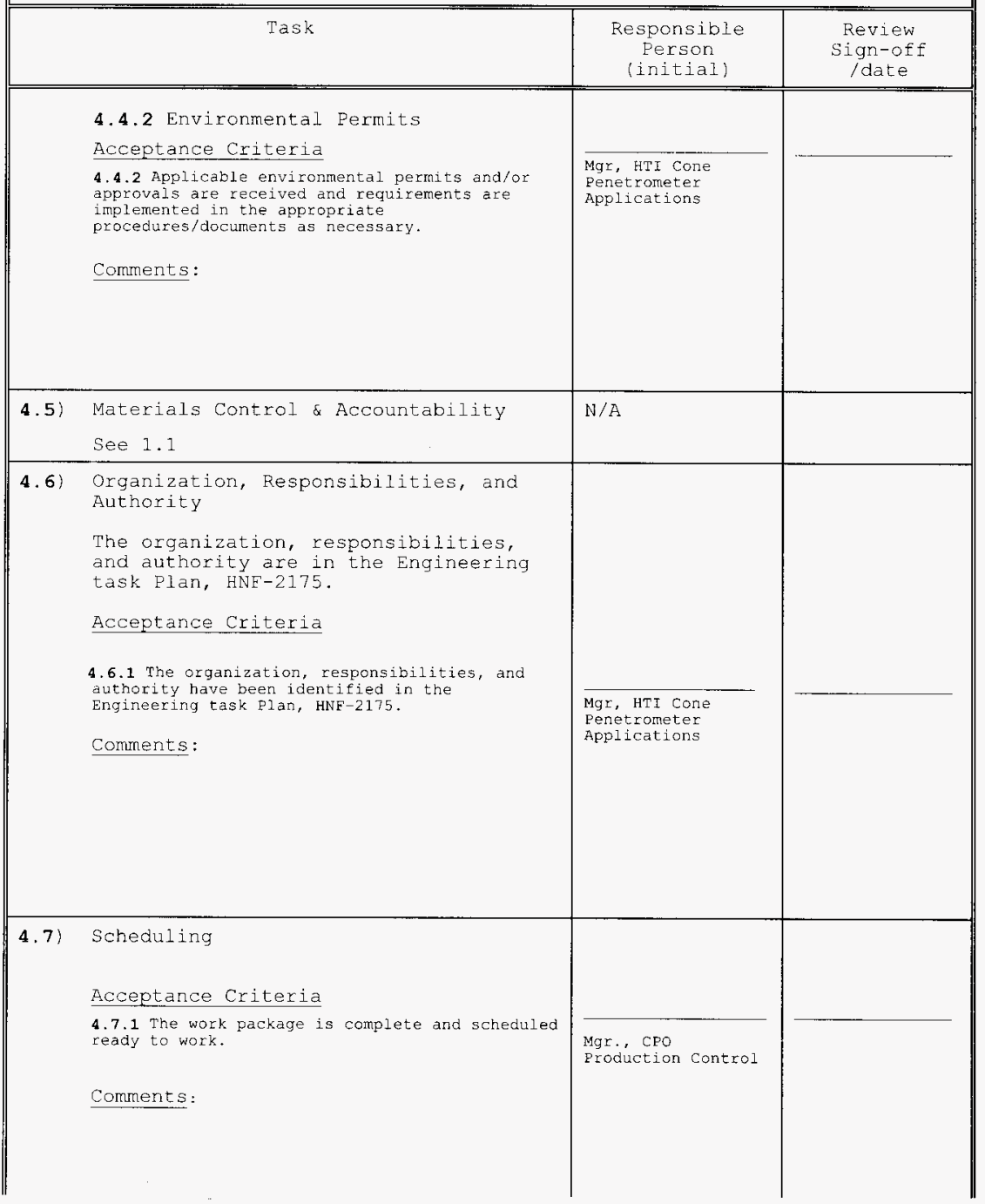




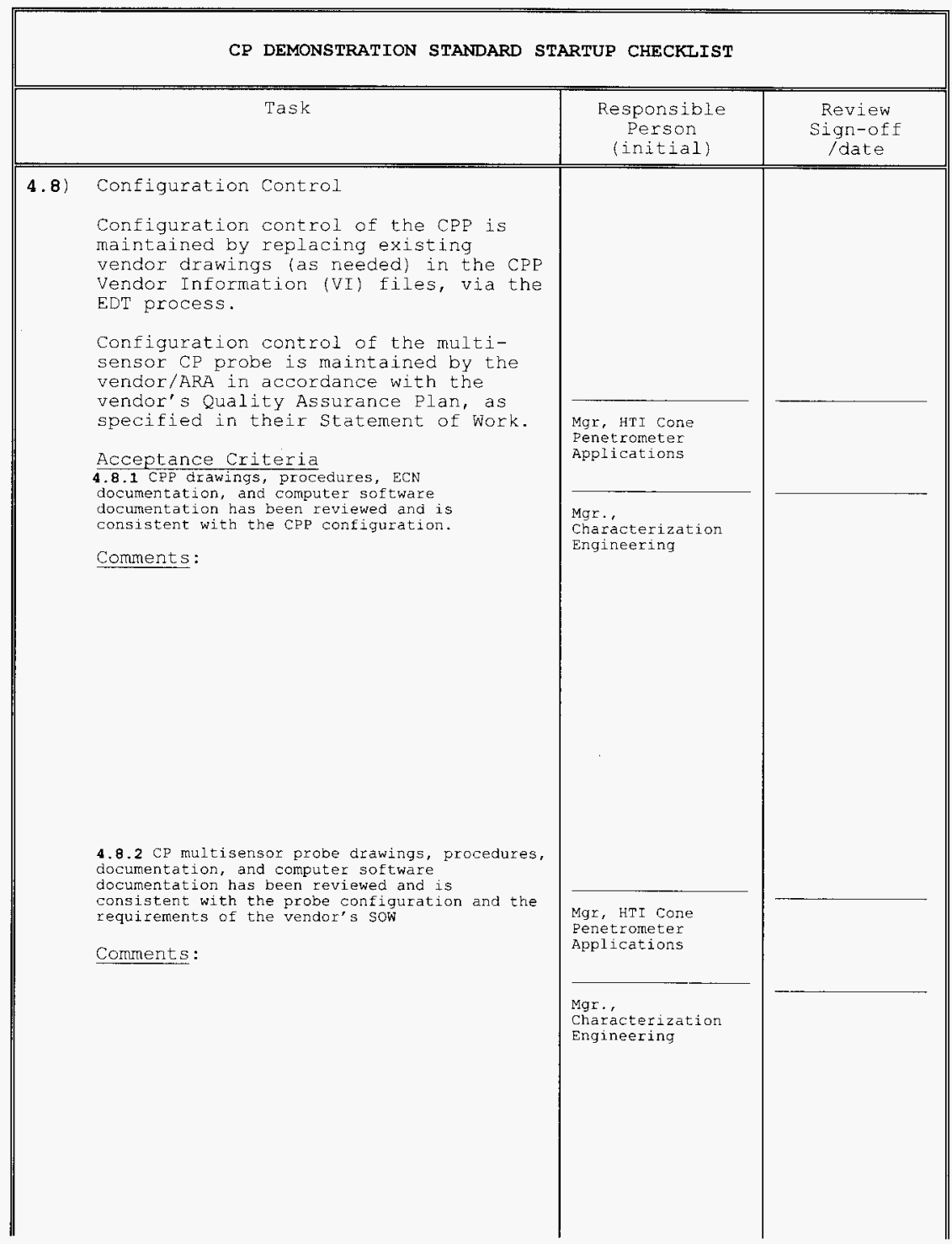




\section{CP DEMONSTRATION STANDARD STARTUP CHECKLIST}

\begin{tabular}{|c|c|c|c|}
\hline & Task & $\begin{array}{c}\text { Responsible } \\
\text { Person } \\
\text { (initial) }\end{array}$ & $\begin{array}{l}\text { Review } \\
\text { Sign-off } \\
\text { /date }\end{array}$ \\
\hline 4.9) & $\begin{array}{l}\text { Technical Control and Data } \\
4.9 .1 \text { Cone Penetrometer Grouting } \\
\text { Strategy Document } \\
\text { Acceptance Criteria } \\
\text { 4.9.1 The Cone Penetrometer Grouting Strategy } \\
\text { Cocument list has been approved and released. } \\
\text { Comments: } \\
\text { 4.9.2 ALARA Management Worksheet } \\
\text { Acceptance Criteria } \\
\text { C.9.2An ALARA Management worksheet has been } \\
\text { Comments: }\end{array}$ & $\begin{array}{l}4.9 .1 \\
\text { Lead Geologist, } \\
\text { Mobilization } \\
\text { Coordinator } \\
4.9 .2 \\
\text { CeP Design Agent, } \\
\text { Deployment } \\
\text { Coordinator } \\
\text { 4.9.2 } \\
\text { Mgr., } \\
\text { Characterization } \\
\text { Field Sampling }\end{array}$ & \\
\hline 4.10$)$ & $\begin{array}{l}\text { Eacility Performance Measurement \& } \\
\text { Evaluation } \\
\text { There are no facility performance } \\
\text { measurements or evaluations associated } \\
\text { with this demonstration. }\end{array}$ & $N / A$ & \\
\hline 4. 11) & $\begin{array}{l}\text { Analysis of Startup of Eacility } \\
\text { Operation and Programmatic Aspects } \\
\text { This cone Penetrometer deployment is a } \\
\text { demonstration not a startup of a } \\
\text { facility. }\end{array}$ & $N / A$ & \\
\hline 4.12$)$ & $\begin{array}{l}\text { Program Definition and Goal } \\
\text { Realization } \\
\text { Acceptance Criteria } \\
4.12 .1 \text { The program definition and goals are } \\
\text { defined in the Hanford Tanks Initiative } \\
\text { Characterization Technical Basis Review (TBR) } \\
\text { (060.020). } \\
\text { Comments: }\end{array}$ & $\begin{array}{l}\text { Mgr, HTI Cone } \\
\text { Penetrometer } \\
\text { Applications }\end{array}$ & \\
\hline
\end{tabular}




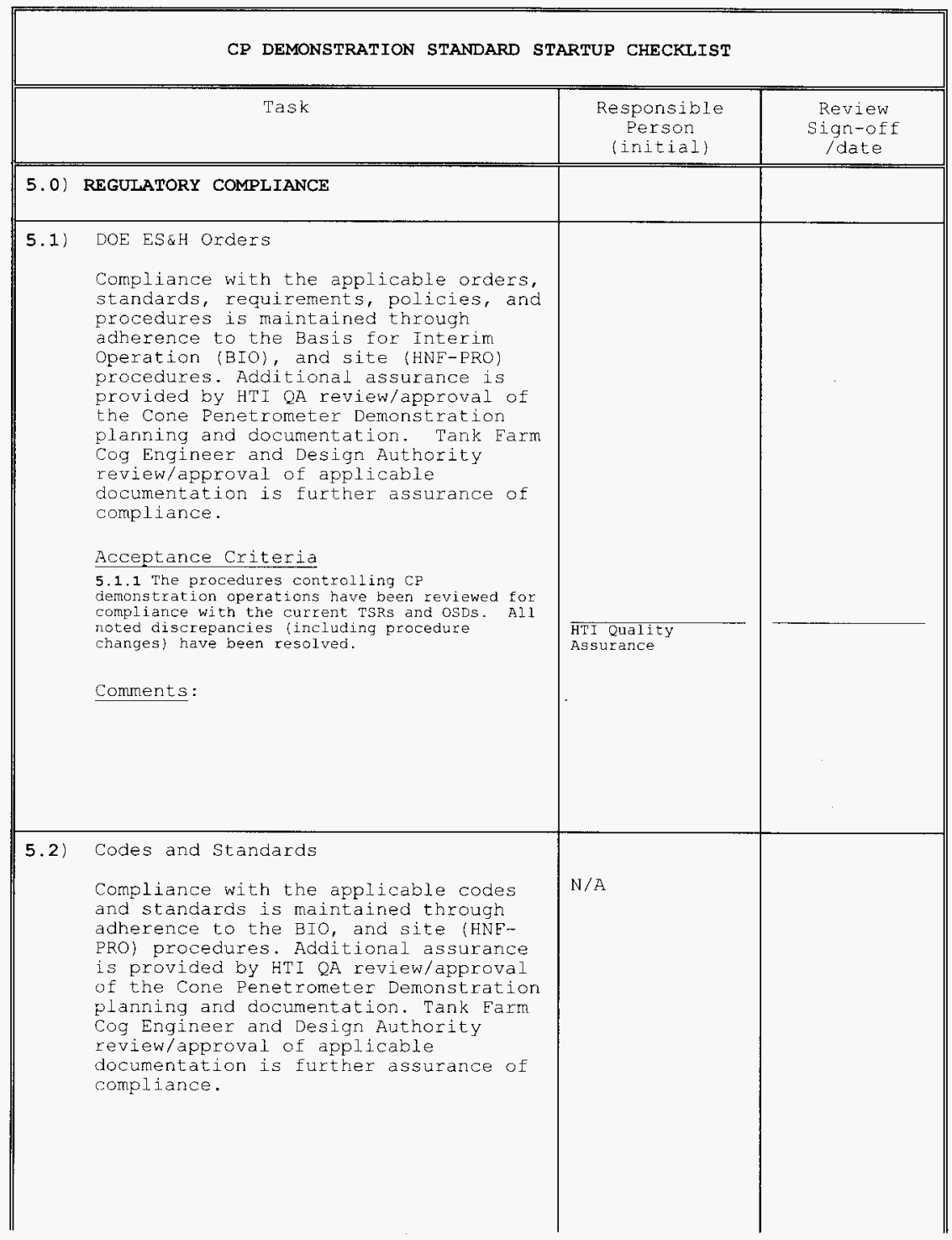




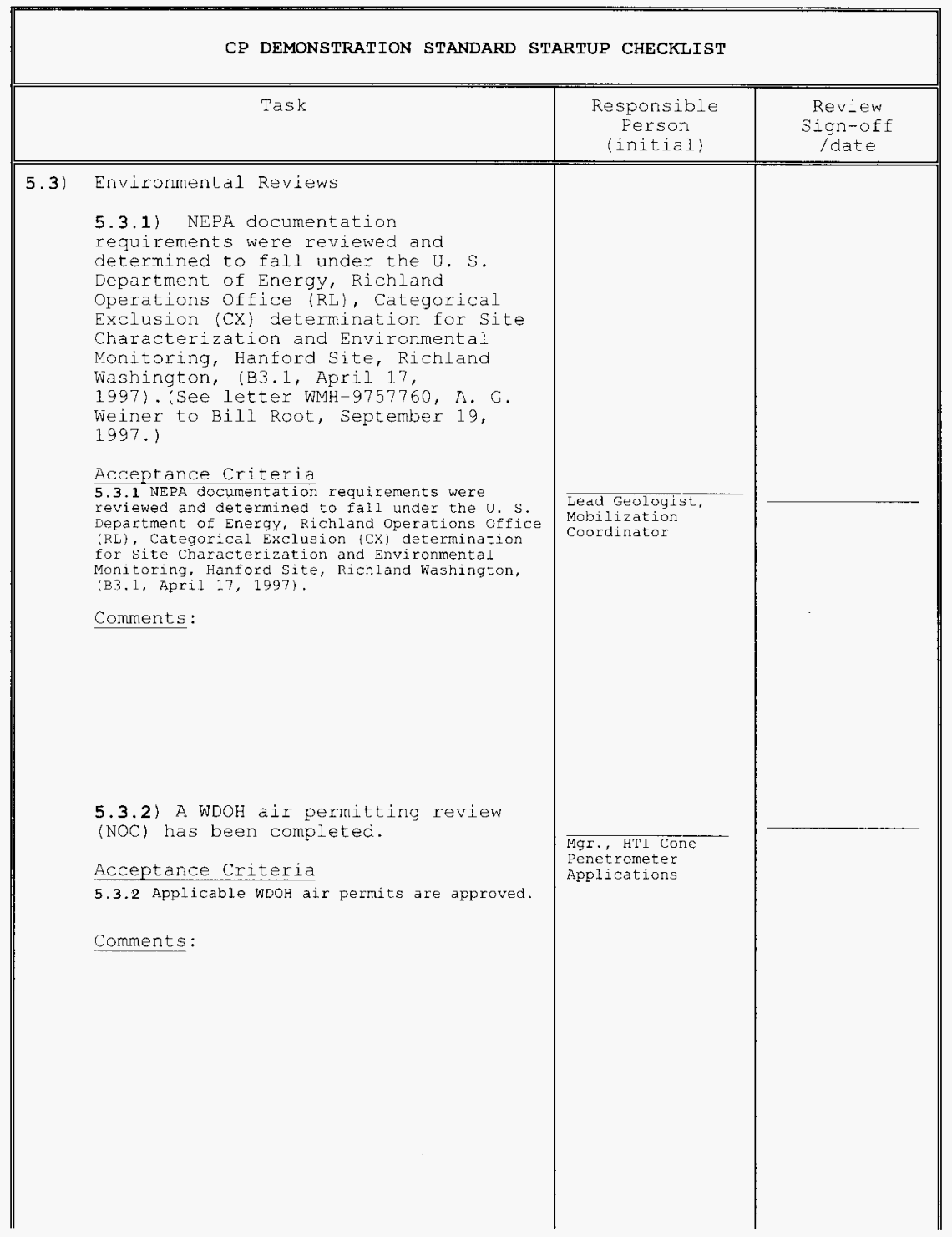




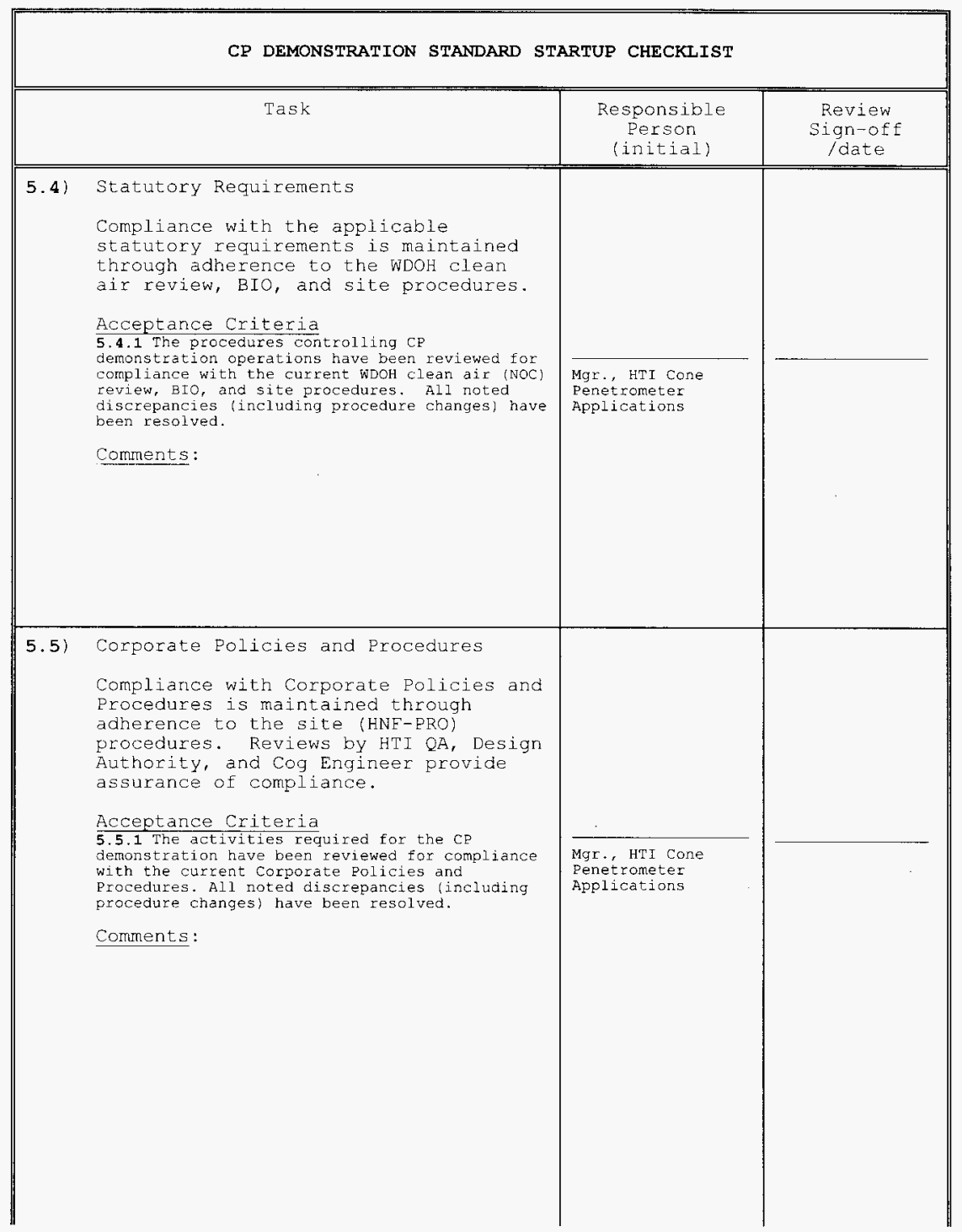




\section{CP DEMONSTRATION STANDARD STARTUP CHECKLIST}

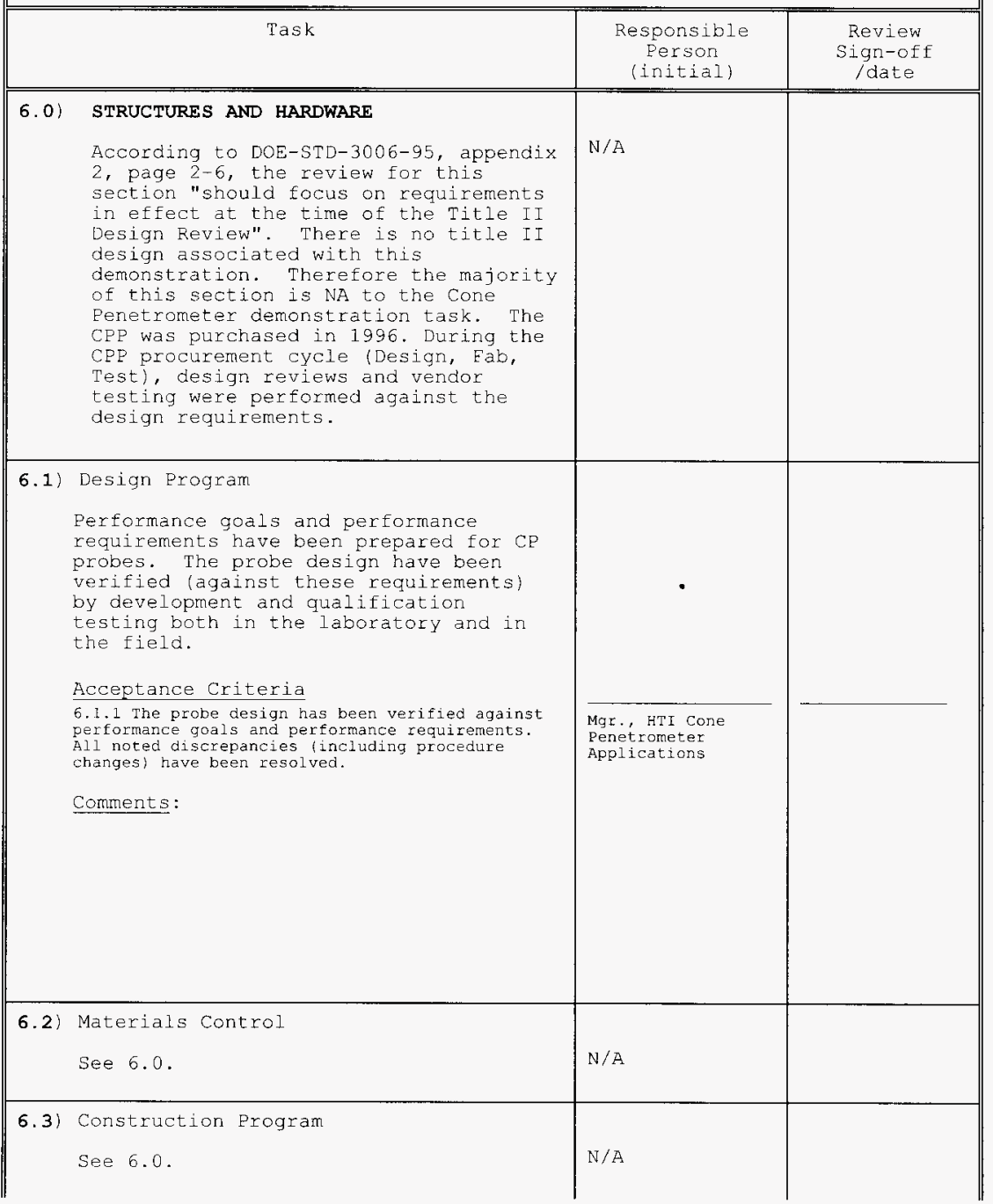


CP DEMONSTRATION STANDARD STARTUP CHECKLIST

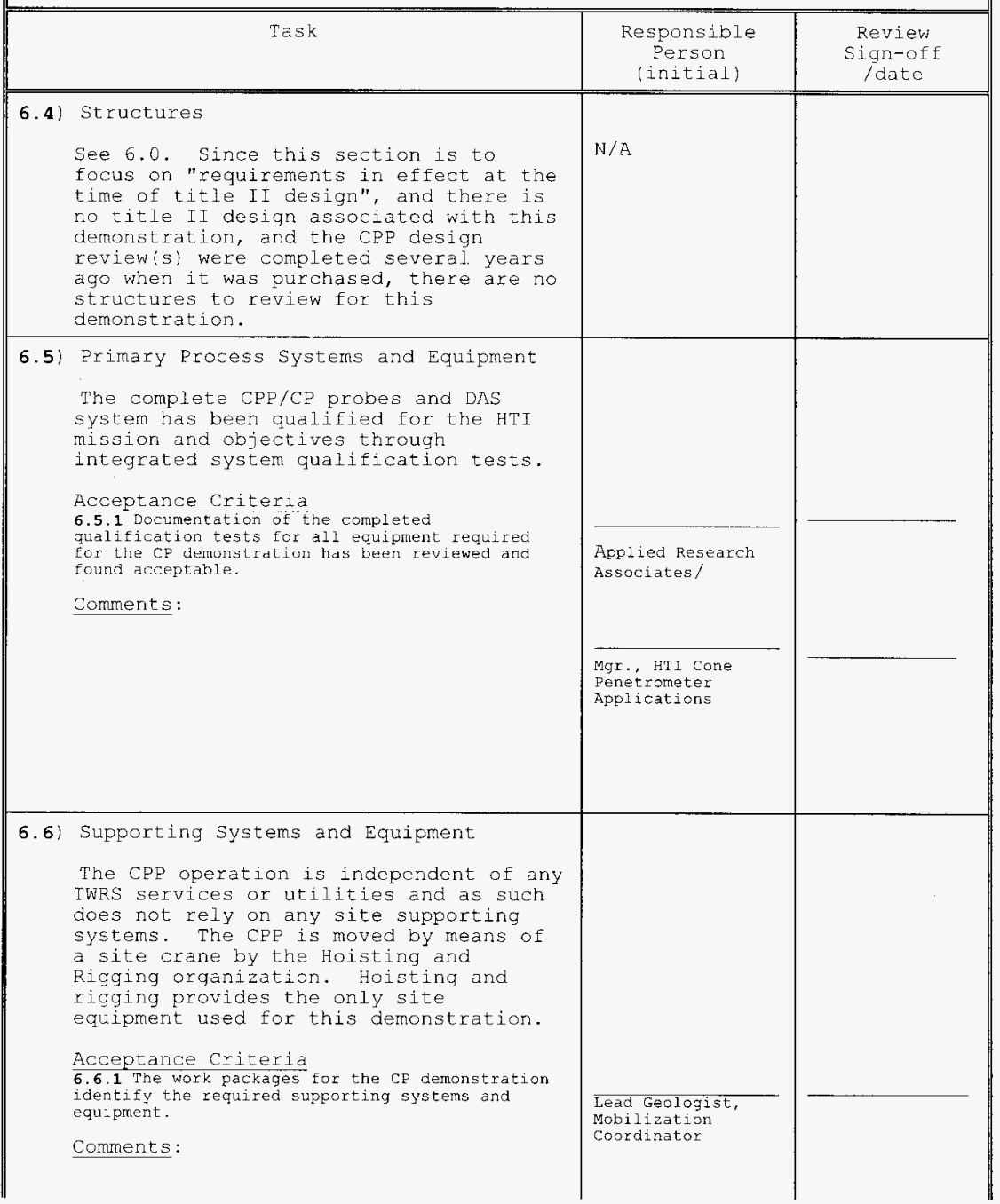




\section{CP DEMONSTRATION STANDARD STARTUP CHECKLIST}

\begin{tabular}{|c|c|c|}
\hline Task & $\begin{array}{c}\text { Responsible } \\
\text { Person } \\
\text { (initial) }\end{array}$ & $\begin{array}{c}\text { Review } \\
\text { Sign-off } \\
\text { /date }\end{array}$ \\
\hline $\begin{array}{l}\text { 6.7) Special Equipment } \\
\text { There is no "special equipment" } \\
\text { associated with this demonstration. } \\
\text { There are performance goals and } \\
\text { performance requirements for the cP } \\
\text { probes. The probe design has been } \\
\text { verified (against these requirements) } \\
\text { developmental and qualification testing } \\
\text { both in the laboratory and in the field } \\
\text { (See } 6.1 \text { above). }\end{array}$ & $N / A$ & \\
\hline $\begin{array}{l}\text { 6.8) Process, Eacility, and site Interfaces } \\
\text { Tank farm interfaces are addressed in } \\
\text { the excavation permit process. } \\
\text { Acceptance_Criteria } \\
\text { 6.B.1 Process, facility, and site interfaces are } \\
\text { identified. } \\
\text { Comments: }\end{array}$ & $\begin{array}{l}\text { Lead Geologist, } \\
\text { Mobilization } \\
\text { Coordinator }\end{array}$ & \\
\hline $\begin{array}{l}\text { 6.9) Materials, spare Parts, and Spare } \\
\text { Equipment } \\
\text { ARA will provide spare probe tips and } \\
\text { pipe sections. } \\
\text { ARA will also provide replacement parts } \\
\text { on an as needed basis for the CPP. } \\
\text { There is no inventory of spare parts } \\
\text { for the CPP. } \\
\text { Acceptance Criteria } \\
\text { 6.9.1 Adequate materials, spare parts, and spare } \\
\text { equipment have been provided for the ce } \\
\text { demonstration. Contract documentation has been } \\
\text { provided regarding ARA's responsibility to } \\
\text { provide needed spare parts. } \\
\text { Comments: }\end{array}$ & $\begin{array}{l}\text { Mgr., HTI Cone } \\
\text { Penetrometer } \\
\text { Applications } \\
\text { Applied Research } \\
\text { Associates }\end{array}$ & \\
\hline
\end{tabular}




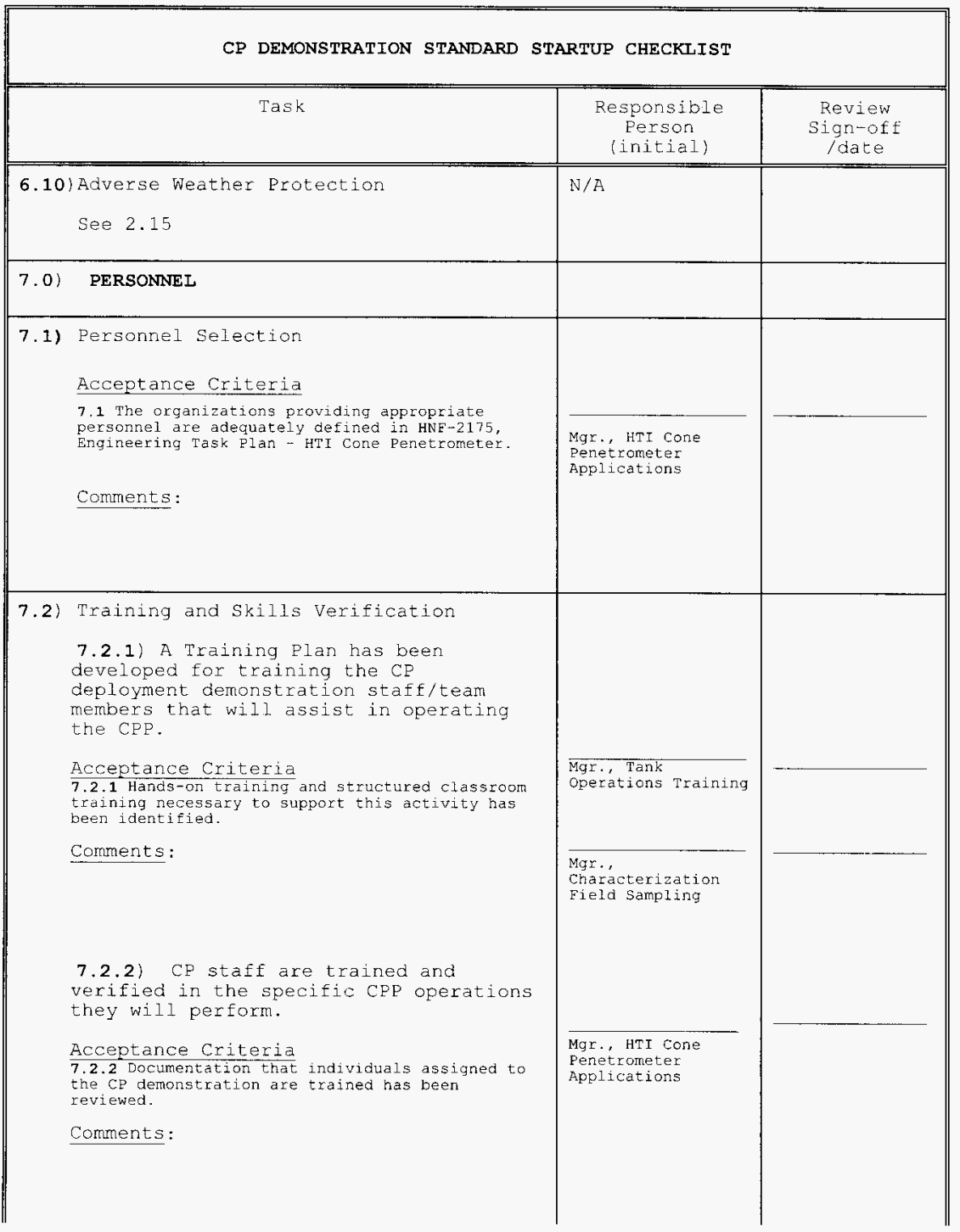




\section{CP DEMONSTRATION STANDARD STARTUP CHECKLIST}

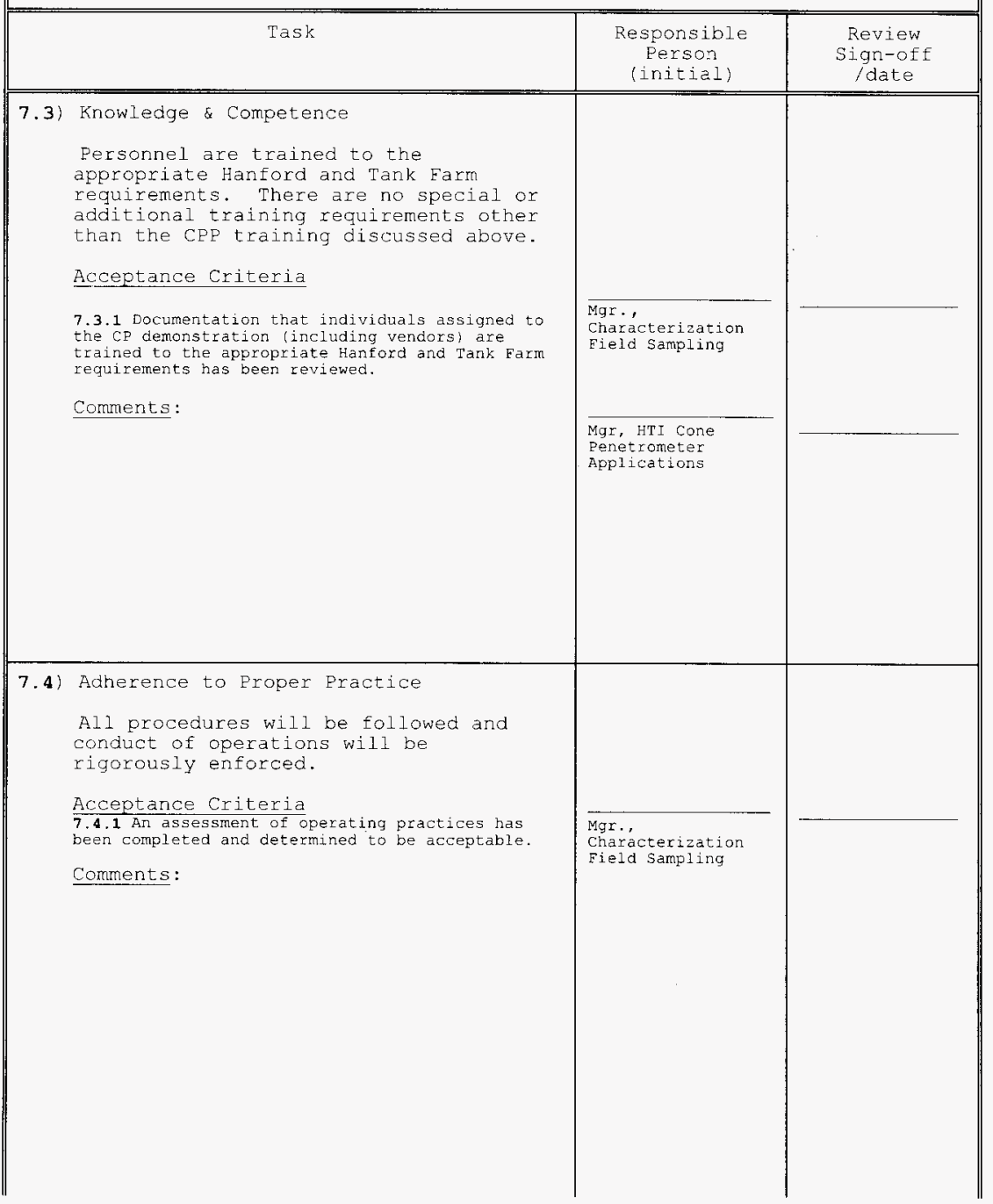




\begin{tabular}{|c|c|c|c|}
\hline \multicolumn{4}{|c|}{ CP DEMONSTRATION STANDARD STARTUP CHECKLIST } \\
\hline & Task & $\begin{array}{l}\text { Responsible } \\
\text { Person } \\
\text { (initial) }\end{array}$ & $\begin{array}{l}\text { Review } \\
\text { Sign-off } \\
\text { /date }\end{array}$ \\
\hline 7.5$)$ & $\begin{array}{l}\text { Staffing Levels } \\
\text { This demonstration is a one-time short- } \\
\text { term operation that does not require } \\
\text { significant staffing. TWRs staffing } \\
\text { requirements (HPT, Supervisor, and } \\
\text { operators) are provided by } \\
\text { Characterization Project Operations. } \\
\text { Acceptance Criteria } \\
\text { 7.5.1 Sufficient trained operating personnel are } \\
\text { demonstration operations will be conducted. } \\
\text { Comments: }\end{array}$ & $\begin{array}{l}\text { Mgr., } \\
\text { Characterization } \\
\text { Field Sampling }\end{array}$ & \\
\hline 7. 6) & $\begin{array}{l}\text { Recertification } \\
\text { There are no recertifications } \\
\text { associated with this demonstration. } \\
\text { Comments: }\end{array}$ & $\mathrm{N} / \mathrm{A}$ & \\
\hline 7.71 & $\begin{array}{l}\text { Oral/Written boards } \\
\text { There are no oral/written boards } \\
\text { associated with this demonstration. }\end{array}$ & $\mathrm{N} / \mathrm{A}$ & \\
\hline 8.0$)$ & OPERATIONS & & \\
\hline 8.1$)$ & $\begin{array}{l}\text { Operations Structure and Management } \\
\text { The roles and responsibilities for this } \\
\text { demonstration are defined in section } \\
4.0 \text { (Roles and Responsibilities) and } \\
\text { depicted in figure } 1 \text { of the Engineering } \\
\text { Task Plan (HNE-2175, Current Rev). }\end{array}$ & $N / A$ & \\
\hline
\end{tabular}




\section{CP DEMONSTRATION STANDARD STARTUP CHECKLIST}

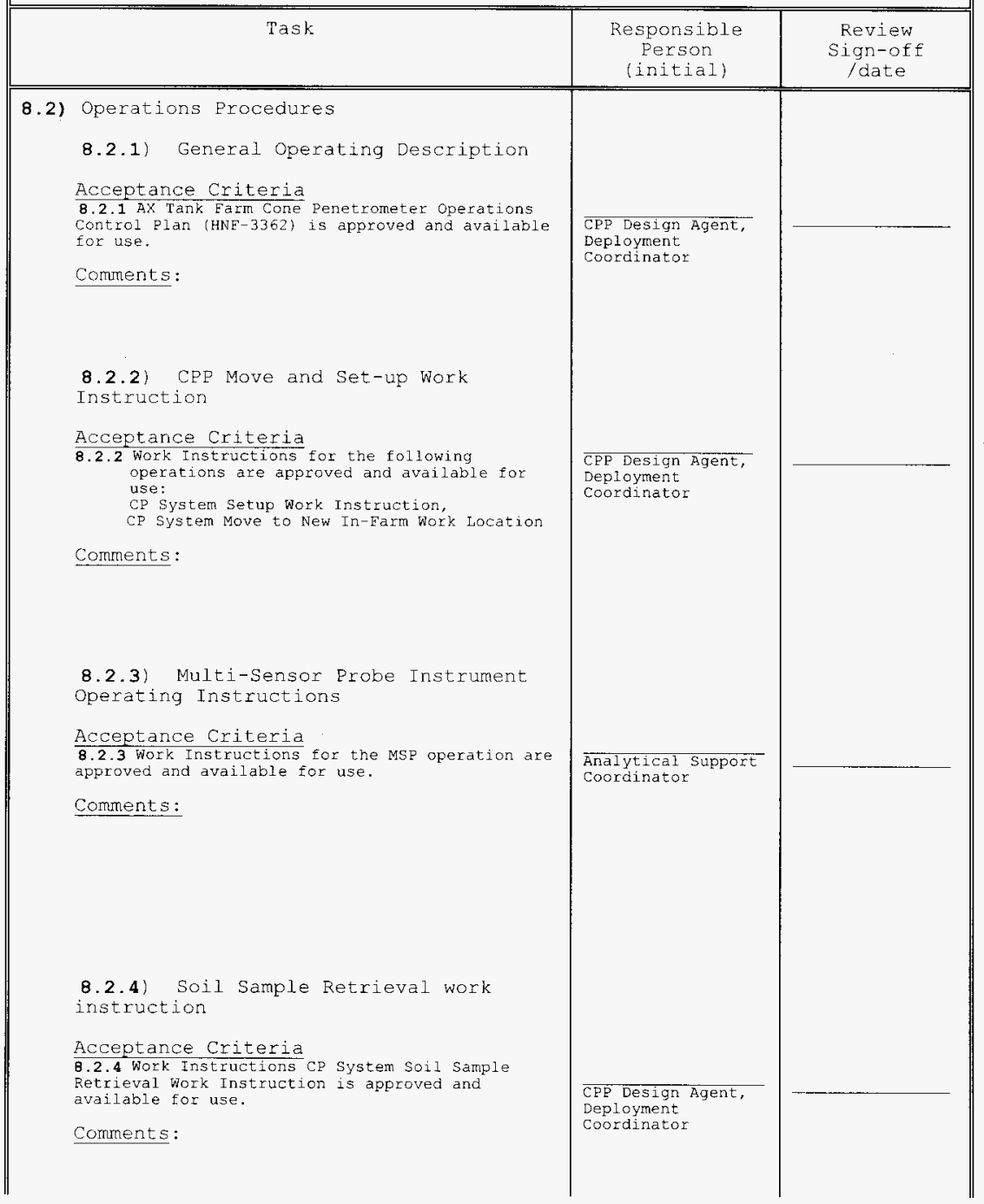




\section{CP DEMONSTRATION STANDARD STARTUP CHECKLIST}

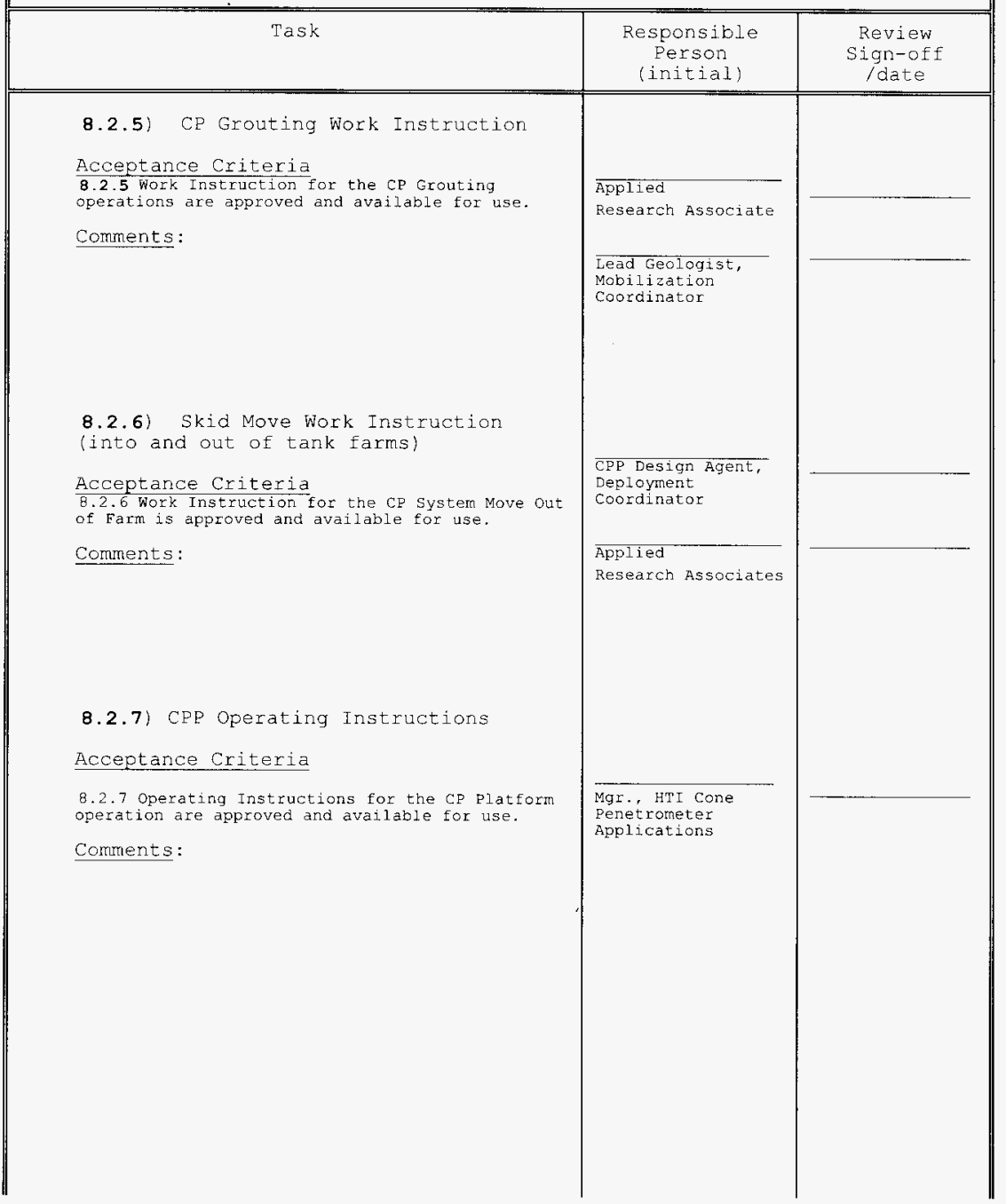




\section{CP DEMONSTRATION STANDARD STARTUP CHECKLIST}

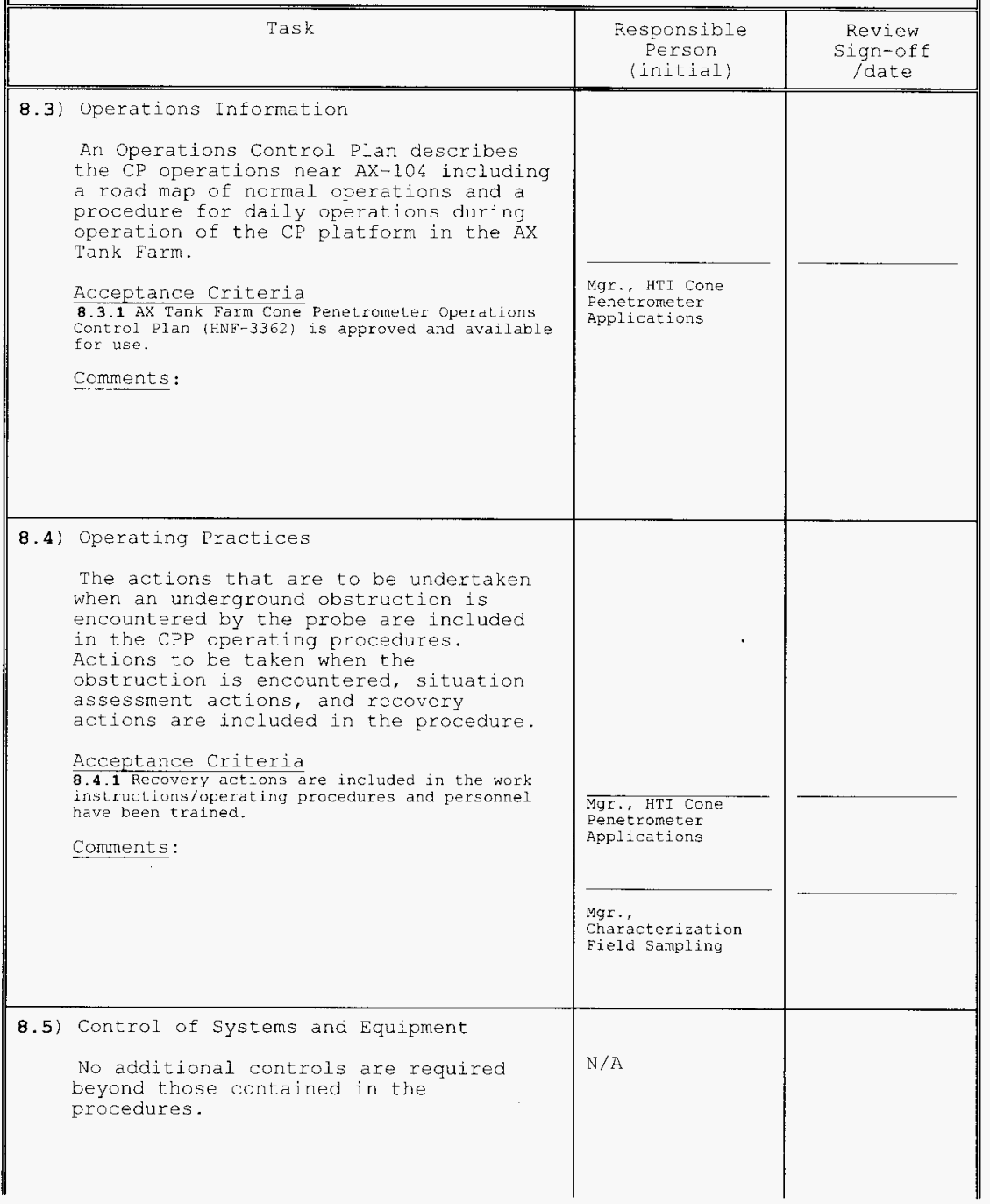




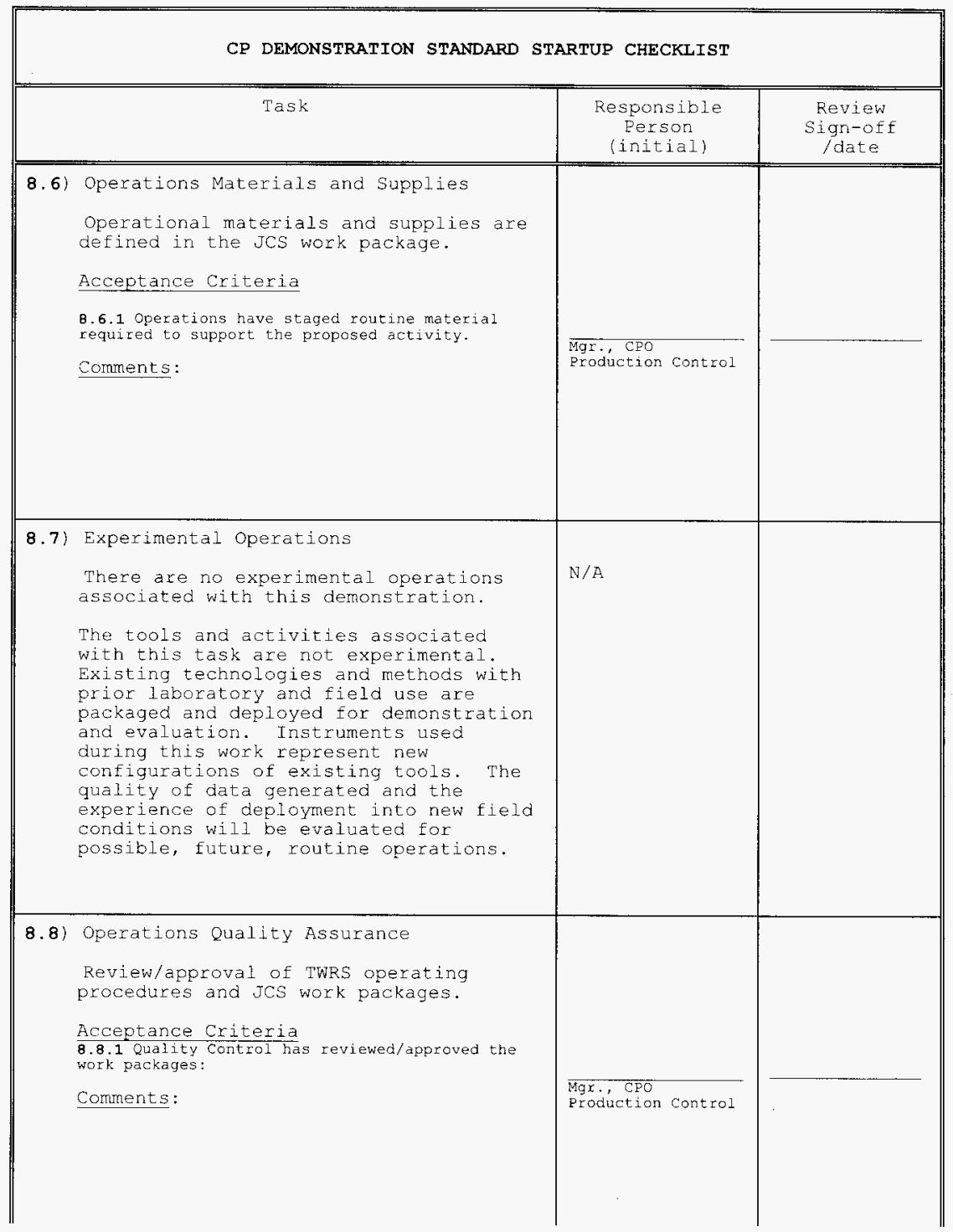


HNE-3101

Revision 1

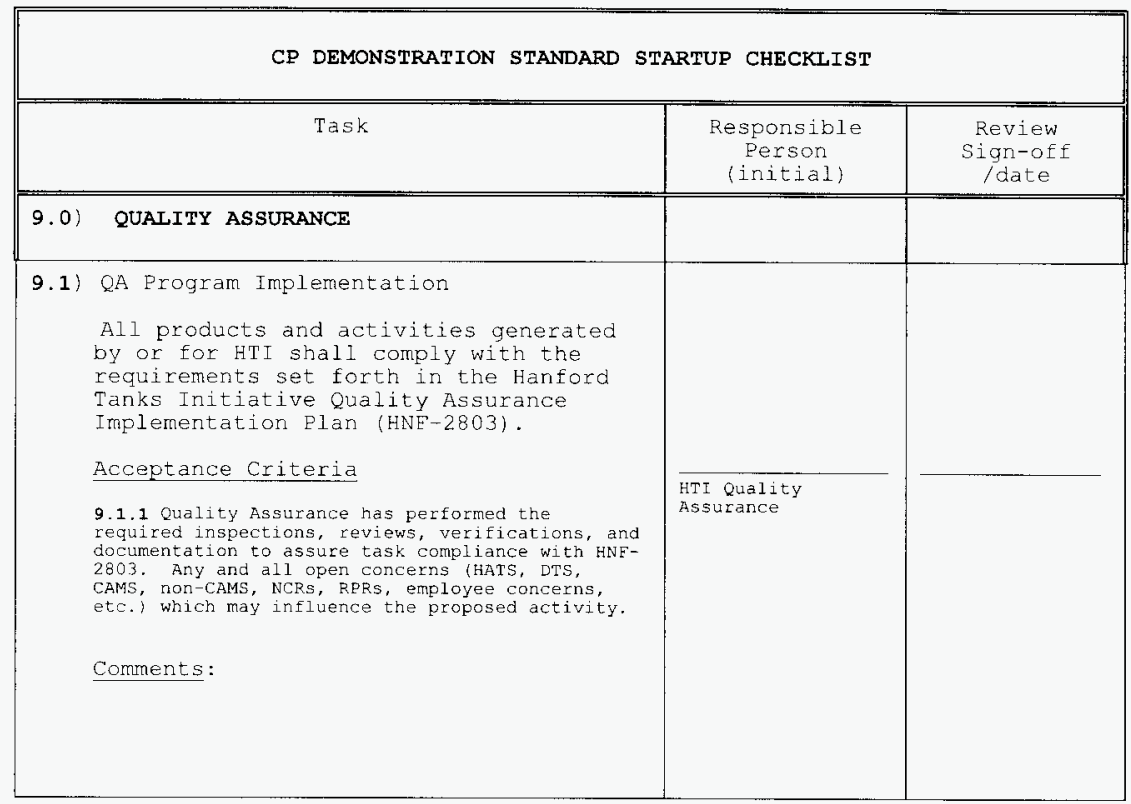

APPROVALS

$\begin{array}{ll}\text { Approval Authority } & \text { Date } \\ \text { Readiness Lead } & \text { Date }\end{array}$




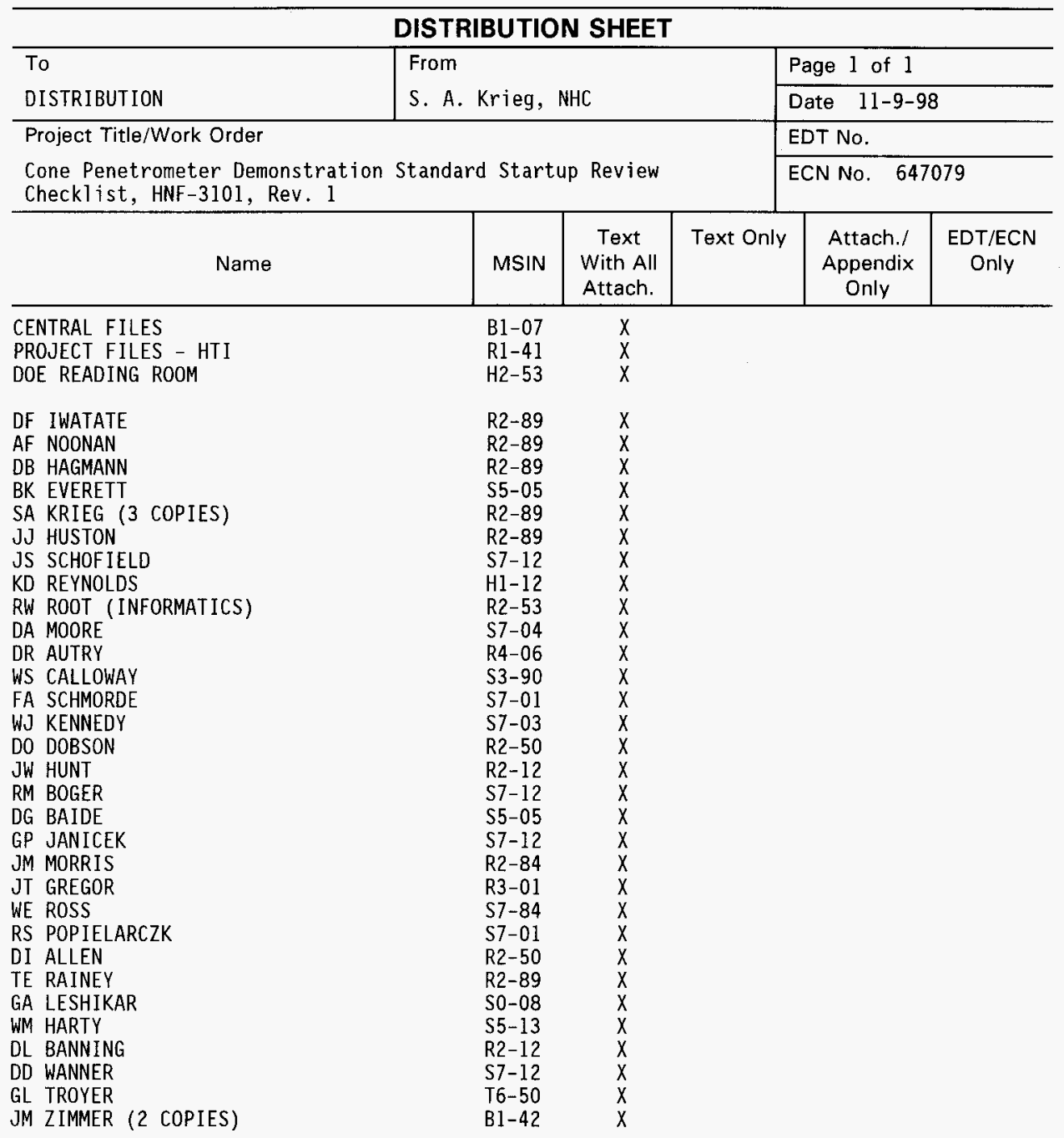

